TRANSACTIONS OF THE

AMERICAN MATHEMATICAL SOCIETY

Volume 361, Number 5, May 2009, Pages 2487-2507

S 0002-9947(08)04573-X

Article electronically published on November 3, 2008

\title{
RESIDUAL $p$ PROPERTIES OF MAPPING CLASS GROUPS AND SURFACE GROUPS
}

\author{
LUIS PARIS
}

\begin{abstract}
Let $\mathcal{M}(\Sigma, \mathcal{P})$ be the mapping class group of a punctured oriented surface $(\Sigma, \mathcal{P})$ (where $\mathcal{P}$ may be empty), and let $\mathcal{T}_{p}(\Sigma, \mathcal{P})$ be the kernel of the action of $\mathcal{M}(\Sigma, \mathcal{P})$ on $H_{1}\left(\Sigma \backslash \mathcal{P}, \mathbb{F}_{p}\right)$. We prove that $\mathcal{T}_{p}(\Sigma, \mathcal{P})$ is residually $p$. In particular, this shows that $\mathcal{M}(\Sigma, \mathcal{P})$ is virtually residually $p$. For a group $G$ we denote by $\mathcal{I}_{p}(G)$ the kernel of the natural action of $\operatorname{Out}(G)$ on $H_{1}\left(G, \mathbb{F}_{p}\right)$. In order to achieve our theorem, we prove that, under certain conditions ( $G$ is conjugacy $p$-separable and has Property A), the group $\mathcal{I}_{p}(G)$ is residually $p$. The fact that free groups and surface groups have Property A is due to Grossman. The fact that free groups are conjugacy $p$-separable is due to Lyndon and Schupp. The fact that surface groups are conjugacy $p$-separable is, from a technical point of view, the main result of the paper.
\end{abstract}

\section{INTRODUCTION}

A group $G$ is said to be residually nilpotent if for every $g \in G \backslash\{1\}$ there exists a homomorphism $\varphi: G \rightarrow N$ such that $N$ is a nilpotent group and $\varphi(g) \neq 1$. For $g, h \in G$, we denote by $[g, h]=g^{-1} h^{-1} g h$ the commutator of $g$ and $h$, and, if $K, H$ are two subgroups of $G$, we denote by $[K, H]$ the commutator subgroup of $K$ and $H$. This is the subgroup generated by $\{[g, h] ; g \in K$ and $h \in H\}$, and it is normal (resp. characteristic) if both $K$ and $H$ are normal (resp. characteristic). By a filtration of a group $G$ we mean an infinite descending chain

$$
G=G_{1} \triangleright G_{2} \triangleright G_{3} \triangleright \cdots \triangleright G_{n} \triangleright \cdots
$$

of normal subgroups. We say that $\left\{G_{n}\right\}_{n=1}^{+\infty}$ is a central filtration if $\left[G_{n}, G_{m}\right] \subset$ $G_{m+n}$ for all $m, n \geq 1$, and we say that $\left\{G_{n}\right\}_{n=1}^{+\infty}$ is a separating filtration if $\bigcap_{n=1}^{+\infty} G_{n}=\{1\}$. It is well-known that a group $G$ is residually nilpotent if and only if it admits a separating central filtration. A central filtration $\left\{G_{n}\right\}_{n=1}^{+\infty}$ is called $\mathbb{Z}$-linear if $G_{n} / G_{n+1}$ is a free $\mathbb{Z}$-module for all $n \geq 1$.

Let $\Sigma_{\rho}$ be an oriented closed surface of genus $\rho \geq 1$, let $\mathcal{P}=\left\{P_{1}, \ldots, P_{n}\right\}$ be a (possibly empty) finite collection of punctures in $\Sigma_{\rho}$, and let $\operatorname{Homeo}\left(\Sigma_{\rho}, \mathcal{P}\right)$ be the group of homeomorphisms $h: \Sigma_{\rho} \rightarrow \Sigma_{\rho}$ which preserve the orientation and such that $h(\mathcal{P})=\mathcal{P}$. The mapping class group of $\left(\Sigma_{\rho}, \mathcal{P}\right)$ is defined to be the group $\mathcal{M}\left(\Sigma_{\rho}, \mathcal{P}\right)=\pi_{0}\left(\operatorname{Homeo}\left(\Sigma_{\rho}, \mathcal{P}\right)\right)$ of isotopy classes of elements of $\operatorname{Homeo}\left(\Sigma_{\rho}, \mathcal{P}\right)$. The natural action of $\mathcal{M}\left(\Sigma_{\rho}, \mathcal{P}\right)$ on $H_{1}\left(\Sigma_{\rho} \backslash \mathcal{P}, \mathbb{Z}\right)$ leads to a homomorphism $\tau$ : $\mathcal{M}\left(\Sigma_{\rho}, \mathcal{P}\right) \rightarrow G L\left(H_{1}\left(\Sigma_{\rho} \backslash \mathcal{P}, \mathbb{Z}\right)\right)$ whose kernel is the Torelli group $\mathcal{T}\left(\Sigma_{\rho}, \mathcal{P}\right)$.

Received by the editors April 2, 2007.

2000 Mathematics Subject Classification. Primary 20F38; Secondary 20E26, 20F14, 20F34, $57 \mathrm{M} 99$.

(C)2008 American Mathematical Society Reverts to public domain 28 years from publication 
The following result is more or less stated in [8] but, as far as I know, its first complete proof is given in [3] (see also 6]).

Theorem 1.1. The Torelli group $\mathcal{T}\left(\Sigma_{\rho}, \mathcal{P}\right)$ has a $\mathbb{Z}$-linear separating central filtration. In particular, $\mathcal{T}\left(\Sigma_{\rho}, \mathcal{P}\right)$ is residually nilpotent.

The filtration of Theorem 1.1 is actually the well-studied Johnson filtration.

Let $\mathcal{F}=\left\{G_{n}\right\}_{n=1}^{+\infty}$ be a central filtration. Then the group commutator induces on

$$
\mathcal{L}_{\mathcal{F}}(G)=\bigoplus_{n=1}^{+\infty} \frac{G_{n}}{G_{n+1}}
$$

a Lie bracket under which it becomes a graded Lie $\mathbb{Z}$-algebra. If, as in Theorem 1.1, the filtration is $\mathbb{Z}$-linear, then $\mathcal{L}_{\mathcal{F}}(G)$ is torsion free and one can apply the Poincaré-Birkhoff-Witt theorem to embed $\mathcal{L}_{\mathcal{F}}(G)$ into its enveloping algebra.

Theorem 1.1 also implies that $\mathcal{T}\left(\Sigma_{\rho}, \mathcal{P}\right)$ is biorderable (see [19]), which implies many other properties (see [17, [5]).

Let $p$ be a prime number. Our goal in this paper is to prove a result analogous to Theorem 1.1, where the Torelli group is replaced by the congruence subgroup $\mathcal{T}_{p}\left(\Sigma_{\rho}, \mathcal{P}\right)$, and the $\mathbb{Z}$-linear filtrations by the $\mathbb{F}_{p}$-linear filtrations.

We say that a group $G$ is residually $p$ if for all $g \in G \backslash\{1\}$ there exists a homomorphism $\varphi: G \rightarrow P$ such that $P$ is a finite $p$-group and $\varphi(g) \neq 1$. Note that residually $p$-groups are residually nilpotent as well as residually finite. A central filtration $\mathcal{F}=\left\{G_{n}\right\}_{n=1}^{+\infty}$ of a group $G$ is called $\mathbb{F}_{p}$-linear if $G_{n} / G_{n+1}$ is a finite dimensional $\mathbb{F}_{p}$-vector space for all $n \geq 1$. Note that, in that case, $\mathcal{L}_{\mathcal{F}}(G)$ becomes a Lie $\mathbb{F}_{p}$-algebra, and, therefore, one can apply the Poincaré-Birkhoff-Witt theorem as well.

Let $\Sigma_{\rho}$ be a closed surface of genus $\rho \geq 1$, and let $\mathcal{P}=\left\{P_{1}, \ldots, P_{n}\right\}$ be a finite collection of punctures in $\Sigma_{\rho}$. The natural action of $\mathcal{M}\left(\Sigma_{\rho}, \mathcal{P}\right)$ on $H_{1}\left(\Sigma_{\rho} \backslash \mathcal{P}, \mathbb{F}_{p}\right)$ leads to a homomorphism $\tau_{p}: \mathcal{M}\left(\Sigma_{\rho}, \mathcal{P}\right) \rightarrow G L\left(H_{1}\left(\Sigma_{\rho} \backslash \mathcal{P}, \mathbb{F}_{p}\right)\right)$ whose kernel is the congruence subgroup $\mathcal{T}_{p}\left(\Sigma_{\rho}, \mathcal{P}\right)$. This is a finite index subgroup of $\mathcal{M}\left(\Sigma_{\rho}, \mathcal{P}\right)$, and is torsion free if $p \neq 2$ (see [10]).

Our main result is the following.

Theorem 1.2. $\mathcal{T}_{p}\left(\Sigma_{\rho}, \mathcal{P}\right)$ is residually $p$.

Remark. We will show in Section 2 (see Proposition 2.3) that a finitely generated group which is residually $p$ admits an $\mathbb{F}_{p}$-linear separating central filtration. On the other hand, $\mathcal{T}_{p}\left(\Sigma_{\rho}, \mathcal{P}\right)$ is a finite index subgroup of $\mathcal{M}\left(\Sigma_{\rho}, \mathcal{P}\right)$ which is finitely generated, thus $\mathcal{T}_{p}\left(\Sigma_{\rho}, \mathcal{P}\right)$ is finitely generated. So, by Theorem 1.2, $\mathcal{T}_{p}\left(\Sigma_{\rho}, \mathcal{P}\right)$ admits an $\mathbb{F}_{p}$-linear separating central filtration.

The proof of Theorem 1.2 is essentially algebraic and uses the following result known as the Dehn-Nielsen-Baer theorem (see [11, 23]).

Theorem 1.3 (Dehn, Nielsen [18, Baer 22, Magnus [15], Zieschang 22]). Let $\Sigma_{\rho}$ be a closed oriented surface of genus $\rho \geq 1$, and let $\mathcal{P}=\left\{P_{1}, \ldots, P_{n}\right\}$ be a finite collection of punctures in $\Sigma_{\rho}$. Then $\mathcal{M}\left(\Sigma_{\rho}, \mathcal{P}\right)$ embeds in $\operatorname{Out}\left(\pi_{1}\left(\Sigma_{\rho} \backslash \mathcal{P}\right)\right)$. Moreover, if $\mathcal{P}=\emptyset$, then $\mathcal{M}\left(\Sigma_{\rho}\right)=\mathcal{M}\left(\Sigma_{\rho}, \emptyset\right)$ is an index 2 subgroup of $\operatorname{Out}\left(\pi_{1}\left(\Sigma_{\rho}\right)\right)$.

For a group $G$, we denote by $I_{p}(G)$ the kernel of the natural homomorphism $\operatorname{Aut}(G) \rightarrow G L\left(H_{1}\left(G, \mathbb{F}_{p}\right)\right)$, and by $\mathcal{I}_{p}(G)$ the kernel of the natural homomorphism $\operatorname{Out}(G) \rightarrow G L\left(H_{1}\left(G, \mathbb{F}_{p}\right)\right)$. Note that $\operatorname{Inn}(G) \subset I_{p}(G)$ and $\mathcal{I}_{p}(G)=I_{p}(G) / \operatorname{Inn}(G)$. Then Theorem 1.2 is a direct consequence of the following. 
Theorem 1.4. Suppose $G$ is either a free group or the fundamental group of a closed oriented surface. Then $I_{p}(G)$ and $\mathcal{I}_{p}(G)$ are both residually $p$.

The fact that the outer automorphism group of a free group is virtually residually $p$ has been previously proved by Lubotzky 13 . He did not give explicitly any finite index subgroup which is residually $p$, but I suspect he knew that $\mathcal{I}_{p}(F)$ is an eligible one. The fact that $I_{p}(G)$ is residually $p$ is more or less known to experts and follows from the following theorem which is proved in Section 2 (see Theorem 2.4).

Theorem 1.5. Let $G$ be a finitely generated and residually $p$ group. Then $I_{p}(G)$ is residually $p$.

Now, in order to prove that $\mathcal{I}_{p}(G)$ is residually $p$ for a given group $G$, we require some restrictions on $G$.

For $g, h \in G$, we use the notation $g \sim h$ to mean that $g$ is conjugate to $h$. We say that a group $G$ is conjugacy p-separable if for all $g, h \in G$, either $g \sim h$ or there exists a homomorphism $\varphi: G \rightarrow P$ such that $P$ is a finite $p$-group and $\varphi(g) \nsim \varphi(h)$. We say that $G$ has Property $A$ if for every automorphism $\alpha \in \operatorname{Aut}(G)$ such that $\alpha(g) \sim g$ for all $g \in G$, we have $\alpha \in \operatorname{Inn}(G)$.

In Section 2 we prove the following.

Theorem 1.6. Let $G$ be a finitely generated group. If $G$ is conjugacy p-separable and has Property $A$, then $\mathcal{I}_{p}(G)$ is residually $p$.

The fact that free groups and fundamental groups of oriented closed surfaces have Property A is proved in [7. The fact that free groups are conjugacy $p$-separable is stated in [14, Proposition 4.8. However, the proof given in 14 is imprecise and incomplete. We give a complete and detailed proof of this result in Section 3. In Section 4 we prove the following.

Theorem 1.7. The fundamental group of a closed oriented surface is conjugacy p-separable.

From a technical point of view, Theorem 1.7 is the main result of the paper.

\section{Automorphism GROUPS AND OUTER AUTOMORPHISM GROUPS OF RESIDUALLY $p$ GROUPS}

We fix a prime number $p \geq 2$. If $K$ and $H$ are two subgroups of a group $G$, we denote by $[K, H]^{p}$ the subgroup generated by $\{[g, h] ; g \in K$ and $h \in H\} \cup\left\{h^{p} ; h \in\right.$ $H\}$. Note that we do not have $[K, H]^{p}=[H, K]^{p}$ in general even if $K$ and $H$ are both normal. The lower $\mathbb{F}_{p}$-linear central filtration of $G$ is defined to be the filtration $\left\{\gamma_{n}^{p} G\right\}_{n=1}^{+\infty}$, where $\gamma_{1}^{p} G=G$ and $\gamma_{n+1}^{p} G=\left[G, \gamma_{n}^{p} G\right]^{p}$ for $n \geq 1$. Note that $G / \gamma_{2}^{p} G=H_{1}\left(G, \mathbb{F}_{p}\right)$.

The term "central" in the above definition can be used because of the following.

Lemma 2.1. Let $n, m \geq 1$. Then $\left[\gamma_{m}^{p} G, \gamma_{n}^{p} G\right] \subset \gamma_{n+m}^{p} G$.

Proof. We argue by induction on $m$. If $m=1$, then

$$
\left[\gamma_{m}^{p} G, \gamma_{n}^{p} G\right]=\left[G, \gamma_{n}^{p} G\right] \subset\left[G, \gamma_{n}^{p} G\right]^{p}=\gamma_{n+1}^{p} G .
$$

Assume $m>1$. We need to prove that $[g, h] \in \gamma_{m+n}^{p} G$ for every generator $g$ of $\gamma_{m}^{p} G$ and every element $h \in \gamma_{n}^{p} G$. There are two different cases to consider: (1) $g \in\left[G, \gamma_{m-1}^{p} G\right] ;(2) g=g_{1}^{p}$ for some $g_{1} \in \gamma_{m-1}^{p} G$. 
Suppose $g \in\left[G, \gamma_{m-1}^{p} G\right]$. By [16, Theorem 5.2, we have

$$
\left.\left[\left[G, \gamma_{m-1}^{p} G\right], \gamma_{n}^{p} G\right] \subset\left[\left[\gamma_{m-1}^{p} G, \gamma_{n}^{p} G\right], G\right] \cdot\left[\gamma_{n}^{p} G, G\right], \gamma_{m-1}^{p} G\right] .
$$

By induction we have

$$
\begin{gathered}
{\left[\left[\gamma_{m-1}^{p} G, \gamma_{n}^{p} G\right], G\right] \subset\left[\gamma_{m+n-1}^{p} G, G\right] \subset \gamma_{m+n}^{p} G,} \\
{\left[\left[\gamma_{n}^{p} G, G\right], \gamma_{m-1}^{p} G\right] \subset\left[\gamma_{n+1}^{p} G, \gamma_{m-1}^{p} G\right] \subset \gamma_{m+n}^{p} G,}
\end{gathered}
$$

thus

$$
[g, h] \in\left[\left[G, \gamma_{m-1}^{p} G\right], \gamma_{n}^{p} G\right] \subset \gamma_{m+n}^{p} G .
$$

Now, we assume that $g=g_{1}^{p}$ for some $g_{1} \in \gamma_{m-1}^{p} G$. Let $g_{2}$ be another element of $\gamma_{m-1}^{p} G$. We have

$$
\left[g_{1} g_{2}, h\right]=\left[g_{1}, h\right] \cdot\left[\left[g_{1}, h\right], g_{2}\right] \cdot\left[g_{2}, h\right]
$$

and, by induction,

$\left[\left[g_{1}, h\right], g_{2}\right] \in\left[\left[\gamma_{m-1}^{p} G, \gamma_{n}^{p} G\right], \gamma_{m-1}^{p} G\right] \subset\left[\gamma_{m+n-1}^{p} G, \gamma_{m-1}^{p} G\right] \subset \gamma_{2 m+n-2}^{p} G \subset \gamma_{m+n}^{p} G$,

thus

$$
\left[g_{1} g_{2}, h\right] \equiv\left[g_{1}, h\right] \cdot\left[g_{2}, h\right] \bmod \gamma_{m+n}^{p} G .
$$

Iterating this congruence we obtain

$$
[g, h]=\left[g_{1}^{p}, h\right] \equiv\left(\left[g_{1}, h\right]\right)^{p} \bmod \gamma_{m+n}^{p} G,
$$

thus $[g, h] \in \gamma_{m+n}^{p} G$ since $\left[g_{1}, h\right] \in\left[\gamma_{m-1}^{p} G, \gamma_{n}^{p} G\right] \subset \gamma_{m+n-1}^{p} G$.

The proof of the following is left to the reader.

Lemma 2.2. Let $P$ be a finitely generated group. Then $P$ is a finite p-group if and only if there exists some $n \geq 1$ such that $\gamma_{n}^{p} P=\{1\}$.

By a $p$-filtration of a group $G$ we mean a filtration $\left\{G_{n}\right\}_{n=1}^{+\infty}$ such that $G / G_{n}$ is a finite $p$-group for all $n \geq 1$. Note that the $p$-filtrations that will appear in the proofs of Theorems 2.4 and 2.5 are actually $\mathbb{F}_{p}$-linear filtrations, but this is not needed for our purpose. Recall also that a filtration $\left\{G_{n}\right\}_{n=1}^{+\infty}$ is said to be a separating filtration if $\bigcap_{n=1}^{+\infty} G_{n}=\{1\}$.

The main criterion we will use in the proofs of Theorems 2.4 and 2.5 are the following.

Proposition 2.3. $\quad$ (1) Let $G$ be a (discrete) group. If $G$ has a separating pfiltration, then $G$ is residually $p$.

(2) Let $G$ be a finitely generated group. Then $G$ is residually $p$ if and only if the lower $\mathbb{F}_{p}$-linear central filtration $\left\{\gamma_{n}^{p} G\right\}_{n=1}^{+\infty}$ is a separating filtration.

Remark. It is easily seen that $\gamma_{n}^{p} G$ is finitely generated for all $n \geq 1$ and that $\left\{\gamma_{n}^{p} G\right\}_{n=1}^{+\infty}$ is a $\mathbb{F}_{p}$-linear filtration if $G$ is finitely generated. This is not necessarily true anymore if $G$ is not finitely generated.

Proof. Part (1) is easy to prove and is left to the reader. So, we assume that $G$ is finitely generated and turn to prove part (2). If $\left\{\gamma_{n}^{p} G\right\}_{n=1}^{+\infty}$ is separating, then $G$ is residually $p$ because $\left\{\gamma_{n}^{p} G\right\}_{n=1}^{+\infty}$ is a $p$-filtration (apply part (1)). Suppose that $G$ is residually $p$. Let $g \in G \backslash\{1\}$. There exists a homomorphism $\varphi: G \rightarrow P$ such that $P$ is a finite $p$-group and $\varphi(g) \neq 1$. By Lemma 2.2, there exists $n \geq 1$ such that $\gamma_{n}^{p} P=\{1\}$. We have $\varphi\left(\gamma_{n}^{p} G\right) \subset \gamma_{n}^{p} P=\{1\}$, thus $g \notin \gamma_{n}^{p} G$ since $\varphi(g) \neq 1$. This shows that $\left\{\gamma_{n}^{p} G\right\}_{n=1}^{+\infty}$ is a separating filtration. 
Theorem 2.4. Let $G$ be a finitely generated group. If $G$ is residually $p$, then $I_{p}(G)$ is residually $p$.

Proof. We consider the lower $\mathbb{F}_{p}$-linear central filtration $\left\{\gamma_{n}^{p} G\right\}_{n=1}^{+\infty}$. Since $\gamma_{n+1}^{p} G$ is a characteristic subgroup of $G$, the quotient map $G \rightarrow G / \gamma_{n+1}^{p} G$ determines a homomorphism

$$
\pi_{n}: \operatorname{Aut} G \rightarrow \operatorname{Aut}\left(G / \gamma_{n+1}^{p} G\right) .
$$

We set $A_{n}=\operatorname{Ker} \pi_{n}$ for all $n \geq 1$ and turn to prove that $\left\{A_{n}\right\}_{n=1}^{+\infty}$ is a separating $p$-filtration of $A_{1}=I_{p}(G)$.

Claim 1. $A_{n}$ acts trivially on $\gamma_{k}^{p} G / \gamma_{n+k}^{p} G$ for all $k \geq 1$.

Proof of Claim 1. We argue by induction on $k$. The case $k=1$ follows from the definition of $A_{n}$, so we can assume $k>1$. We take $\alpha \in A_{n}$, and turn to prove that $\alpha(g) \equiv g \bmod \gamma_{n+k}^{p} G$ for every generator $g$ of $\gamma_{k}^{p} G$.

Suppose first that $g=\left[g_{1}, h\right]$ for some $g_{1} \in \gamma_{k-1}^{p} G$ and $h \in G$. By induction, there exist $x_{1} \in \gamma_{k+n-1}^{p} G$ and $y \in \gamma_{n+1}^{p} G$ such that $\alpha\left(g_{1}\right)=g_{1} x_{1}$ and $\alpha(h)=h y$. By Lemma 2.1,

$$
\begin{gathered}
{\left[g_{1} x_{1}, y\right] \in\left[\gamma_{k-1}^{p} G, \gamma_{n+1}^{p} G\right] \subset \gamma_{k+n}^{p} G} \\
{\left[\left[g_{1} x_{1}, h\right], y\right] \in\left[\left[\gamma_{k-1}^{p} G, G\right], \gamma_{n+1}^{p} G\right] \subset\left[\gamma_{k}^{p} G, \gamma_{n+1}^{p} G\right] \subset \gamma_{k+n+1}^{p} G \subset \gamma_{k+n}^{p} G,}
\end{gathered}
$$

thus

$$
\alpha(g)=\left[g_{1} x_{1}, h y\right]=\left[g_{1} x_{1}, h\right] \cdot\left[g_{1} x_{1}, y\right] \cdot\left[\left[g_{1} x_{1}, h\right], y\right] \equiv\left[g_{1} x_{1}, h\right] \bmod \gamma_{k+n}^{p} G .
$$

Again, by Lemma 2.1,

$$
\begin{gathered}
{\left[\left[g_{1}, h\right], x_{1}\right] \in\left[\left[\gamma_{k-1}^{p} G, G\right], \gamma_{k+n-1}^{p} G\right] \subset\left[\gamma_{k}^{p} G, \gamma_{k+n-1}^{p} G\right] \subset \gamma_{2 k+n-1}^{p} G \subset \gamma_{k+n}^{p} G,} \\
{\left[x_{1}, h\right] \in\left[\gamma_{k+n-1}^{p} G, G\right] \subset \gamma_{k+n}^{p} G,}
\end{gathered}
$$

thus

$$
\left[g_{1} x_{1}, h\right]=\left[g_{1}, h\right] \cdot\left[\left[g_{1}, h\right], x_{1}\right] \cdot\left[x_{1}, h\right] \equiv\left[g_{1}, h\right]=g \bmod \gamma_{k+n}^{p} G .
$$

This shows that $\alpha(g) \equiv g \bmod \gamma_{k+n}^{p} G$.

Now, we assume that $g=g_{1}^{p}$ for some $g_{1} \in \gamma_{k-1}^{p} G$. Let $g_{2}$ be another element of $\gamma_{k-1}^{p} G$. By induction, there exist $x_{1}, x_{2} \in \gamma_{k+n-1}^{p} G$ such that $\alpha\left(g_{1}\right)=g_{1} x_{1}$ and $\alpha\left(g_{2}\right)=g_{2} x_{2}$. By Lemma 2.1,

$$
\left.\left[g_{2}, x_{1}^{-1}\right] \in\left[\gamma_{k-1}^{p} G, \gamma_{k+n-1}^{p} G\right] \subset \gamma_{2 k+n-2}^{p} G \subset \gamma_{k+n}^{p} G \quad \text { (since } k \geq 2\right),
$$

thus

$$
\alpha\left(g_{1} g_{2}\right)=g_{1} x_{1} g_{2} x_{2}=g_{1} g_{2}\left[g_{2}, x_{1}^{-1}\right] x_{1} x_{2} \equiv g_{1} g_{2} x_{1} x_{2} \bmod \gamma_{k+n}^{p} G .
$$

Iterating the above congruence we obtain

$$
\alpha(g)=\alpha\left(g_{1}^{p}\right) \equiv g_{1}^{p} x_{1}^{p} \equiv g_{1}^{p} \bmod \gamma_{k+n}^{p} G .
$$

Claim 2. The filtration $\left\{A_{n}\right\}_{n=1}^{+\infty}$ is a separating filtration.

Proof of Claim 2. Let $\alpha \in I_{p}(G), \alpha \neq \mathrm{Id}$. Choose $g \in G$ such that $\alpha(g) \neq g$. Since $\left\{\gamma_{n}^{p} G\right\}_{n=1}^{+\infty}$ is a separating filtration, there exists $n \geq 1$ such that $g^{-1} \alpha(g) \notin \gamma_{n+1}^{p} G$; that is, $\alpha(g) \not \equiv g \bmod \gamma_{n+1}^{p} G$. This shows that $\alpha \notin A_{n}$. 
Claim 3. Let $\alpha \in A_{n}$. Then the map

$$
\begin{aligned}
u_{\alpha}: G / \gamma_{n+1}^{p} G & \rightarrow \gamma_{n+1}^{p} G / \gamma_{n+2}^{p} G, \\
{[g] } & \mapsto\left[g^{-1} \alpha(g)\right]
\end{aligned}
$$

is well-defined.

Proof of Claim 3. Let $g_{1}, g_{2} \in G$ such that $g_{1} \equiv g_{2} \bmod \gamma_{n+1}^{p} G$. Let $x_{1}, x_{2} \in$ $\gamma_{n+1}^{p} G$ such that $\alpha\left(g_{1}\right)=g_{1} x_{1}$ and $\alpha\left(g_{2}\right)=g_{2} x_{2}$. We have to prove that $x_{1} \equiv$ $x_{2} \bmod \gamma_{n+2}^{p} G$. Set $a=g_{1}^{-1} g_{2} \in \gamma_{n+1}^{p} G$. By Claim 1, $\alpha(a)$ is of the form $\alpha(a)=a y$, where $y \in \gamma_{2 n+1}^{p} G \subset \gamma_{n+2}^{p} G$. We have

$$
\left[a, x_{1}^{-1}\right] \in\left[\gamma_{n+1}^{p} G, \gamma_{n+1}^{p} G\right] \subset \gamma_{2 n+2}^{p} G \subset \gamma_{n+2}^{p} G
$$

and

thus

$$
\alpha\left(g_{2}\right)=g_{1} x_{1} a y=g_{1} a\left[a, x_{1}^{-1}\right] x_{1} y=g_{2}\left[a, x_{1}^{-1}\right] x_{1} y,
$$

$$
x_{2}=\left[a, x_{1}^{-1}\right] x_{1} y \equiv x_{1} \bmod \gamma_{n+2}^{p} G .
$$

We denote by $H_{n}$ the set of set-maps from $G / \gamma_{n+1}^{p} G$ to $\gamma_{n+1}^{p} G / \gamma_{n+2}^{p} G$. The group $\gamma_{n+1}^{p} G / \gamma_{n+2}^{p} G$ is a finite dimensional $\mathbb{F}_{p}$-vector space and $G / \gamma_{n+1}^{p} G$ is finite, thus $H_{n}$ is finite and naturally endowed with a structure of $\mathbb{F}_{p}$-vector space. The next claim shows that $A_{n} / A_{n+1}$ is also a finite dimensional $\mathbb{F}_{p}$-vector space for all $n \geq 1$, so it implies that $\left\{A_{n}\right\}_{n=1}^{+\infty}$ is a $p$-filtration.

Claim 4. The map

$$
\begin{aligned}
u: A_{n} & \rightarrow H_{n} \\
\alpha & \mapsto u_{\alpha}
\end{aligned}
$$

is a group homomorphism whose kernel is $A_{n+1}$.

Proof of Claim 4. Let $\alpha_{1}, \alpha_{2} \in A_{n}$ and let $g \in G$. We write $\alpha_{1}(g)=g x_{1}, \alpha_{2}(g)=$ $g x_{2}$, and $\alpha_{2}\left(x_{1}\right)=x_{1} y$, where $x_{1}, x_{2} \in \gamma_{n+1}^{p} G$ and $y \in \gamma_{2 n+1}^{p} G \subset \gamma_{n+2}^{p} G$. We have $\left(\alpha_{2} \alpha_{1}\right)(g)=g x_{2} x_{1} y$, thus

$$
u_{\alpha_{2} \alpha_{1}}([g])=\left[x_{2} x_{1} y\right]=\left[x_{2} x_{1}\right]=u_{\alpha_{2}}([g]) \cdot u_{\alpha_{1}}([g])=\left(u_{\alpha_{2}} \cdot u_{\alpha_{1}}\right)([g]) .
$$

This shows that $u$ is a homomorphism.

Let $\alpha \in A_{n+1}$. If $g \in G$, then $\alpha(g) \equiv g \bmod \gamma_{n+2}^{p} G$, thus $u_{\alpha}([g])=[1]$. This shows that $u_{\alpha}$ is trivial; that is, $\alpha$ belongs to the kernel of $u$.

Let $\alpha$ be an element of the kernel of $u$. Let $g \in G$, and let $x=g^{-1} \alpha(g)$. We have $u_{\alpha}([g])=[x]=[1]$, thus $x \in \gamma_{n+2}^{p} G$, hence $\alpha(g) \equiv g \bmod \gamma_{n+2}^{p} G$. This shows that $\alpha \in A_{n+1}$.

Theorem 2.5. Let $G$ be a finitely generated group. If $G$ is conjugacy p-separable and has Property $A$, then $\mathcal{I}_{p}(G)$ is residually $p$.

Proof. Recall that $\mathcal{I}_{p}(G)=I_{p}(G) / \operatorname{Inn}(G)$. So, in order to prove Theorem 2.5, it suffices to find a $p$-filtration $\left\{B_{n}\right\}_{n=1}^{+\infty}$ of $I_{p}(G)$ such that $\bigcap_{n=1}^{+\infty} B_{n}=\operatorname{Inn}(G)$.

We denote by $\pi_{n}: I_{p}(G) \rightarrow \operatorname{Aut}\left(G / \gamma_{n+1}^{p} G\right)$ the restriction of $\pi_{n}: \operatorname{Aut}(G) \rightarrow$ $\operatorname{Aut}\left(G / \gamma_{n+1}^{p} G\right)$ to $I_{p}(G)$. The kernel of $\pi_{n}$ is the group $A_{n}$ defined in the proof of Theorem 2.4, and $\left\{A_{n}\right\}_{n=1}^{+\infty}$ is a separating $p$-filtration of $I_{p}(G)$. Let $S_{n}$ be the set of conjugacy classes in $G / \gamma_{n+1}^{p} G$, and let $\operatorname{Sym}\left(S_{n}\right)$ denote the symmetric group of $S_{n}$. The group $\operatorname{Aut}\left(G / \gamma_{n+1}^{p} G\right)$ acts naturally on $S_{n}$, and this action defines a homomorphism $\theta_{n}: \operatorname{Aut}\left(G / \gamma_{n+1}^{p} G\right) \rightarrow \operatorname{Sym}\left(S_{n}\right)$. We set $\tilde{\pi}_{n}=\theta_{n} \circ \pi_{n}: I_{p}(G) \rightarrow$ $\operatorname{Sym}\left(S_{n}\right)$, and $B_{n}=\operatorname{Ker} \tilde{\pi}_{n}$. 
Claim 1. $\left\{B_{n}\right\}_{n=1}^{+\infty}$ is a $p$-filtration.

Proof of Claim 1. The fact that $\left\{A_{n}\right\}_{n=1}^{+\infty}$ is a $p$-filtration means that $I_{p}(G) / A_{n} \simeq$ $\operatorname{Im} \pi_{n}$ is a finite $p$-group, thus $I_{p}(G) / B_{n} \simeq \operatorname{Im} \tilde{\pi}_{n}=\theta_{n}\left(\operatorname{Im} \pi_{n}\right)$ is also a finite $p$-group (it is the image of a finite $p$-group under a homomorphism).

Claim 2. $\bigcap_{n=1}^{+\infty} B_{n}=\operatorname{Inn}(G)$.

Proof of Claim 2. The inclusion $\operatorname{Inn}(G) \subset \bigcap_{n=1}^{+\infty} B_{n}$ is clear, thus we only have to prove the reverse inclusion: $\bigcap_{n=1}^{+\infty} B_{n} \subset \operatorname{Inn}(G)$. Let $\alpha \in I_{p}(G), \alpha \notin \operatorname{Inn}(G)$. Since $G$ has Property A, there exists $g \in G$ such that $g$ is not conjugate to $\alpha(g)$. Since $G$ is conjugacy $p$-separable, there exists a homomorphism $\varphi: G \rightarrow P$ such that $P$ is a finite $p$-group and $\varphi(g) \nsim \varphi(\alpha(g))$. By Lemma 2.2, there exists $n \geq 1$ such that $\gamma_{n+1}^{p} P=\{1\}$. We have $\varphi\left(\gamma_{n+1}^{p} G\right) \subset \gamma_{n+1}^{p} P=\{1\}$, thus $\gamma_{n+1}^{p} G \subset \operatorname{Ker} \varphi$. Let $\mu_{n}: G \rightarrow G / \gamma_{n+1}^{p} G$ be the quotient map. The above inclusion and the fact that $\varphi(g) \nsim \varphi(\alpha(g))$ imply that $\mu_{n}(g) \nsim \mu_{n}(\alpha(g))$. This shows that $\tilde{\pi}_{n}(\alpha)$ does not fix the conjugacy class of $[g] \in G / \gamma_{n+1}^{p} G$, thus $\tilde{\pi}_{n}(\alpha) \neq \mathrm{Id}$, therefore $\alpha \notin B_{n}$.

\section{Conjugacy $p$-Separability of Free Groups}

We start with an observation that the reader must keep in mind because it will be often used in the remainder of the paper.

Lemma 3.1. Let $G$ be a group, and let $K_{1}, K_{2}$ be two normal subgroups such that $G / K_{1}$ and $G / K_{2}$ are finite p-groups. Then $G /\left(K_{1} \cap K_{2}\right)$ is a finite p-group.

Proof. Let $\varphi_{1}: G \rightarrow G / K_{1}$ and $\varphi_{2}: G \rightarrow G / K_{2}$ be the quotient maps. Then $K_{1} \cap$ $K_{2}$ is the kernel of the homomorphism $\varphi: G \rightarrow G / K_{1} \times G / K_{2}, g \mapsto\left(\varphi_{1}(g), \varphi_{2}(g)\right)$.

Now, we prove the following.

Theorem 3.2. Free groups are conjugacy p-separable.

Proof. Let $F=F(X)$ be a free group freely generated by a set $X$. Let $g=x_{1}^{\varepsilon_{1}} \cdots x_{l}^{\varepsilon_{l}}$ be an element of $F$ written in normal form (i.e. $x_{i} \in X, \varepsilon_{i} \in\{ \pm 1\}$, and $x_{i+1}^{\varepsilon_{i+1}} \neq x_{i}^{-\varepsilon_{i}}$ for all $i$ ). The number $l$ is called the word length of $g$ and is denoted by $|g|_{X}$, and the set $\left\{x_{1}, \ldots, x_{l}\right\} \subset X$ is called the support of $g$ and is denoted by $\operatorname{Sup}(g)$.

We take two elements $g, h \in F$ that are not conjugate, and we prove by induction on $|g|_{X}+|h|_{X}$ that there exists a homomorphism $\varphi: F \rightarrow P$ such that $P$ is a finite $p$-group and $\varphi(g) \nsim \varphi(h)$.

Step 1. We assume $g=1$.

We have $h \neq 1$ and $F$ is residually $p$ (see [4], for example); thus there exists a homomorphism $\varphi: F \rightarrow P$ such that $P$ is a finite $p$-group and $\varphi(h) \neq 1$. We obviously have $\varphi(h) \nsim \varphi(g)=1$.

Conclusion of Step 1 . We can assume $g \neq 1$ and $h \neq 1$.

Step 2. We assume that $|\operatorname{Sup}(g)|=|\operatorname{Sup}(h)|=1$.

Then $g$ and $h$ are of the form $g=x^{n}$ and $h=y^{m}$, where $x, y \in X$ and $n, m \in$ $\mathbb{Z} \backslash\{0\}$. Moreover, $n \neq m$ if $x=y$. Let $q=p^{e}$ be a power of $p$ strictly greater than $2|n|$ and than $2|m|$. Set $P=\mathbb{Z} / q \mathbb{Z}$, and let $\varphi: F \rightarrow P$ be the homomorphism defined by $\varphi(x)=1$, and $\varphi(z)=0$ for all $z \in X \backslash\{x\}$. We have $\varphi(g) \neq \varphi(h)$, thus $\varphi(g) \not \varphi(h)$ since $P$ is abelian. 
Conclusion of Step 2. We can assume that $|\operatorname{Sup}(g)| \geq 2$.

For $f \in F$, we denote by $[f]_{1}$ the homology class of $f$ in $H_{1}(F)=\bigoplus_{x \in X} \mathbb{Z}[x]_{1}$.

Step 3. We assume that $[g]_{1} \neq[h]_{1}$.

Write

$$
[g]_{1}-[h]_{1}=\sum_{i=1}^{l} a_{i}\left[x_{i}\right]_{1},
$$

where $x_{1}, \ldots, x_{l} \in X$ and $a_{i} \in \mathbb{Z} \backslash\{0\}$. Let $q=p^{e}$ be a power of $p$ strictly greater than $2\left|a_{1}\right|$. Set $P=\mathbb{Z} / q \mathbb{Z}$, and let $\varphi: F \rightarrow P$ be the homomorphism defined by $\varphi\left(x_{1}\right)=1$, and $\varphi(z)=0$ for all $z \in X \backslash\left\{x_{1}\right\}$. We have $\varphi(g) \neq \varphi(h)$, thus $\varphi(g) \nsim \varphi(h)$.

Conclusion of Step 3. We can assume that $[g]_{1}=[h]_{1}$.

Step 4. We prove that there exist a homomorphism $\mu: F \rightarrow \mathbb{Z} / p \mathbb{Z}$ and an element $x \in \operatorname{Sup}(g)$ such that $\mu(x)=1$ and $\mu(g)=\mu(h)=0$.

Suppose $[g]_{1}=[h]_{1}=0$. We choose some $x \in \operatorname{Sup}(g)$ and we define $\mu: F \rightarrow$ $\mathbb{Z} / p \mathbb{Z}$ by $\mu(x)=1$, and $\mu(z)=0$ for all $z \in X \backslash\{x\}$. We have $\mu(g)=\mu(h)=0$.

Suppose $[g]_{1}=[h]_{1}=a[y]_{1}$ for some $y \in X$ and $a \in \mathbb{Z} \backslash\{0\}$. Take $x \in \operatorname{Sup}(g) \backslash\{y\}$ (this is possible because $|\operatorname{Sup}(g)| \geq 2$ ) and define $\mu: F \rightarrow \mathbb{Z} / p \mathbb{Z}$ by $\mu(x)=1$, and $\mu(z)=0$ for all $z \in X \backslash\{x\}$. Then $\mu(g)=\mu(h)=0$.

Suppose $[g]_{1}=[h]_{1}=a_{1}\left[y_{1}\right]_{1}+\cdots+a_{l}\left[y_{l}\right]_{1}$, where $y_{1}, \ldots, y_{l} \in X, a_{1}, \ldots, a_{l} \in$ $\mathbb{Z} \backslash\{0\}$, and $l \geq 2$. Let $m=\operatorname{gcd}\left(a_{1}, \ldots, a_{l}\right), b_{i}=a_{i} / m$ for $1 \leq i \leq l$, and $T_{1}=b_{1}\left[y_{1}\right]_{1}+\cdots+b_{l}\left[y_{l}\right]_{1}$. Let $U=\mathbb{Z}\left[y_{1}\right]_{1} \oplus \cdots \oplus \mathbb{Z}\left[y_{l}\right]_{1}$ be the $\mathbb{Z}$-module freely generated by $\left[y_{1}\right]_{1}, \ldots,\left[y_{l}\right]_{1}$. Since $\operatorname{gcd}\left(b_{1}, \ldots, b_{l}\right)=1, T_{1}$ can be completed into a $\mathbb{Z}$-basis $\left\{T_{1}, T_{2}, \ldots, T_{l}\right\}$ of $U$. We define $\bar{\mu}: H_{1}(F) \rightarrow \mathbb{Z} / p \mathbb{Z}$ by

$$
\begin{gathered}
\bar{\mu}\left(T_{1}\right)=0, \quad \bar{\mu}\left(T_{2}\right)=1, \quad \bar{\mu}\left(T_{i}\right)=0 \text { for } 3 \leq i \leq l, \\
\text { and } \bar{\mu}(z)=0 \text { for } z \in X \backslash\left\{y_{1}, \ldots, y_{l}\right\} .
\end{gathered}
$$

The fact that $\bar{\mu}\left(T_{1}\right)=0$ implies that $\bar{\mu}\left([g]_{1}\right)=\bar{\mu}\left([h]_{1}\right)=0$, and the fact that $\bar{\mu}\left(T_{2}\right) \neq 0$ implies that there exists $x \in\left\{y_{1}, \ldots, y_{l}\right\} \subset \operatorname{Sup}(g)$ such that $\bar{\mu}\left([x]_{1}\right) \neq 0$. Each element of $(\mathbb{Z} / p Z) \backslash\{0\}$ generates $\mathbb{Z} / p \mathbb{Z}$, thus one can find an automorphism $\xi: \mathbb{Z} / p \mathbb{Z} \rightarrow \mathbb{Z} / p \mathbb{Z}$ such that $\xi\left(\bar{\mu}\left([x]_{1}\right)\right)=1$. Set $\mu=\xi \circ \bar{\mu} \circ \kappa: F \rightarrow \mathbb{Z} / p \mathbb{Z}$, where $\kappa: F \rightarrow H_{1}(F)$ is the quotient map. Then $\mu(x)=1$ and $\mu(g)=\mu(h)=0$.

Now, for $y \in X \backslash\{x\}$ and $0 \leq i \leq p-1$, we set

$$
d(y, i)=x^{i} y x^{-\mu\left(x^{i} y\right)} .
$$

Then $\operatorname{Ker} \mu$ is the free group $F(Y)$ freely generated by

$$
Y=\{d(y, i) ; 0 \leq i \leq p-1 \text { and } y \in X \backslash\{x\}\} \cup\left\{x^{p}\right\} .
$$

For $f \in \operatorname{Ker} \mu=F(Y)$ and $0 \leq i \leq p-1$, we set $f_{i}=x^{i} f x^{-i} \in F(Y)$.

Step 5. Let $f \in F(Y)$. We show that $\left|f_{i}\right|_{Y} \leq|f|_{X}$ for all $0 \leq i \leq p-1$, and that, if $x \in \operatorname{Sup}(f)$, then there exists $i \in\{0,1, \ldots, p-1\}$ such that $\left|f_{i}\right|_{Y}<|f|_{X}$.

Set

$$
d(x, i)=\left\{\begin{array}{ll}
1 & \text { if } 0 \leq i \leq p-2, \\
x^{p} & \text { if } i=p-1,
\end{array} \quad d\left(x^{-1}, i\right)= \begin{cases}x^{-p} & \text { if } i=0 \\
1 & \text { if } 1 \leq i \leq p-1\end{cases}\right.
$$


For $y \in X \backslash\{x\}$ and $0 \leq i \leq p-1$ we set

$$
d\left(y^{-1}, i\right)=x^{i} y^{-1} x^{-\mu\left(x^{i} y^{-1}\right)} .
$$

Note that

$$
d\left(y^{-1}, i\right)=d\left(y, \mu\left(x^{i} y^{-1}\right)\right)^{-1}
$$

for all $y \in X$ (included $y=x$ ) and all $0 \leq i \leq p-1$.

Let $f=y_{1}^{\varepsilon_{1}} \cdots y_{l}^{\varepsilon_{l}}$ be the normal form of $f$ with respect to $X$. For $1 \leq j \leq l$ we set

Then

$$
i_{j}=\mu\left(x^{i} y_{1}^{\varepsilon_{1}} \cdots y_{j-1}^{\varepsilon_{j-1}}\right) .
$$

$$
f_{i}=d\left(y_{1}^{\varepsilon_{1}}, i_{1}\right) \cdot d\left(y_{2}^{\varepsilon_{2}}, i_{2}\right) \cdots d\left(y_{l}^{\varepsilon_{l}}, i_{l}\right) .
$$

This shows that $\left|f_{i}\right|_{Y} \leq|f|_{X}$.

Suppose, moreover, that some $y_{j}$ is equal to $x$. We can choose $i \in\{0,1, \ldots, p-1\}$ so that $i_{j} \neq p-1$ if $\varepsilon_{j}=1$, or $i_{j} \neq 0$ if $\varepsilon_{j}=-1$. In this case $d\left(y_{j}^{\varepsilon_{j}}, i_{j}\right)=1$ and, therefore, $\left|f_{i}\right|_{Y}<|f|_{X}$.

Conclusion of Step 5. We can assume that $\left|g_{i_{0}}\right|_{Y}<|g|_{X}$ for some $0 \leq i_{0} \leq p-1$, and $\left|h_{i}\right|_{Y} \leq|h|_{X}$ for all $0 \leq i \leq p-1$.

By the inductive hypothesis, there exists a homomorphism $\alpha_{i}: F(Y) \rightarrow A_{i}$ such that $A_{i}$ is a finite $p$-group and $\alpha_{i}\left(g_{i_{0}}\right) \not \alpha_{i}\left(h_{i}\right)$ for all $0 \leq i \leq p-1$. Let $L=\bigcap_{i=0}^{p-1} \operatorname{Ker} \alpha_{i}$, let $B=F(Y) / L$, and let $\beta: F(Y) \rightarrow B$ be the quotient map. Then $B$ is a finite $p$-group (by Lemma 3.1) and $\beta\left(g_{i_{0}}\right) \nsim \beta\left(h_{i}\right)$ for all $0 \leq i \leq p-1$. Let $K=\bigcap_{i=0}^{p-1} x^{i} L x^{-i}$. It is easily seen that $K$ is a normal subgroup of $F=F(X)$ and that $P=F(X) / K$ is a finite $p$-group. We denote by $\varphi: F \rightarrow P$ the quotient map.

Step 6. We prove that $\varphi(g) \nsim \varphi(h)$.

Suppose not. Then there exists $f \in F(X)$ such that $\varphi\left(g_{i_{0}}\right)=\varphi\left(f h f^{-1}\right)$. Write $f$ in the form $f=f^{\prime} x^{i}$, where $f^{\prime} \in F(Y)$ and $0 \leq i \leq p-1$. Then $\varphi\left(g_{i_{0}}\right)=$ $\varphi\left(f^{\prime} h_{i} f^{\prime-1}\right)$ which implies that $\beta\left(g_{i_{0}}\right)=\beta\left(f^{\prime} h_{i} f^{\prime-1}\right)$, a contradiction.

\section{Conjugacy $p$-Separability of Surface groups}

In this section we prove the following.

Theorem 4.1. The fundamental group of a closed oriented surface of genus $\rho \geq 1$ is conjugacy p-separable.

We fix a genus $\rho \geq 1$, and we denote by $\Sigma_{\rho}$ the oriented closed surface of genus $\rho$. If $\rho=1$, then $\pi_{1}\left(\Sigma_{\rho}\right) \simeq \mathbb{Z}^{2}$, which is obviously conjugacy $p$-separable; thus we can and will assume $\rho \geq 2$.

We use in our proof the fact that $\pi_{1}\left(\Sigma_{\rho}\right)$ can be expressed (in many ways) as the amalgamated product of two free groups along a cyclic group. So, we start with some preliminaries on amalgamated products of groups.

Let $G=H_{1} *_{C} H_{2}$ be the amalgamated product of two groups $H_{1}, H_{2}$ along a subgroup $C$. A normal form of an element $g \in G \backslash C$ is defined to be an expression

$$
g=g_{1} \cdot g_{2} \cdots g_{l}
$$

of $g$ such that

- either $g_{i} \in H_{1} \backslash C$ or $g_{i} \in H_{2} \backslash C$, for all $1 \leq i \leq l$, 
- if $g_{i} \in H_{1} \backslash C$, then $g_{i+1} \in H_{2} \backslash C$, and if $g_{i} \in H_{2} \backslash C$, then $g_{i+1} \in H_{1} \backslash C$, for all $1 \leq i \leq l-1$.

Such an expression always exists but is not unique in general. However, the length $l$ of the normal form is unique. It is called the Syllable length of $g$ and is denoted by $\operatorname{Syl}(g)$. A normal form is said to be cyclically reduced if its length is either 1 or even. Note that in the latest case (when $l$ is even) we have the equivalences

$$
g_{l} \in H_{2} \backslash C \Leftrightarrow g_{1} \in H_{1} \backslash C, \quad g_{l} \in H_{1} \backslash C \Leftrightarrow g_{1} \in H_{2} \backslash C .
$$

If a normal form of $g$ is cyclically reduced, then all its normal forms are cyclically reduced. We say in that case that $g$ itself is cyclically reduced. We also use the convention that the elements of $C$ are cyclically reduced and their syllable lengths are equal to 0 . It is easily checked that every element of $G$ is conjugate to a cyclically reduced element.

We refer to 20] for the proof of the following proposition and for a general exposition on amalgamated products of groups.

Proposition 4.2. Let $G=H_{1} *_{C} H_{2}$ be the amalgamated product of two groups $H_{1}, H_{2}$ along a subgroup $C$, and let $g, h \in G$ be two cyclically reduced elements.

(1) If $g \sim h$, then $\operatorname{Syl}(g)=\operatorname{Syl}(h)$.

(2) Suppose $\operatorname{Syl}(g)=\operatorname{Syl}(h)=l \geq 2$, and let $h=h_{1} \cdots h_{l}$ be a normal form of $h$. We have $g \sim h$ if and only if there exist $u \in C$ and $1 \leq t \leq l$ such that

$$
u g u^{-1}=h_{t} \cdot h_{t+1} \cdots h_{l} \cdot h_{1} \cdots h_{t-1} .
$$

(3) Suppose $\operatorname{Syl}(g)=\operatorname{Syl}(h)=l \geq 2$, and let $g=g_{1} \cdots g_{l}$ and $h=h_{1} \cdots h_{l}$ be normal forms of $g$ and $h$, respectively. There exists $u \in C$ such that $u g u^{-1}=h$ if and only if there exist $l+1$ elements $u_{0}, u_{1}, \ldots, u_{l} \in C$ such that $u_{0}=u_{l}$ and $u_{i-1} g_{i}=h_{i} u_{i}$ for all $1 \leq i \leq l$.

An element $g$ of a group $G$ is said to be conjugacy p-distinguished if, for every $h \in G$, either $h \sim g$ or there exists a homomorphism $\varphi: G \rightarrow P$ such that $P$ is a finite $p$-group and $\varphi(g) \nsim \varphi(h)$. Note that a group $G$ is conjugacy $p$-separable if and only if all its elements are conjugacy $p$-distinguished.

The strategy we use to prove Theorem 4.1 is the following. We start with an element $g \in \pi_{1}\left(\Sigma_{\rho}\right) \backslash\{1\}$ and show that there exists a decomposition $\pi_{1}\left(\Sigma_{\rho}\right)=$ $F_{1} *_{C} F_{2}$ of $\pi_{1}\left(\Sigma_{\rho}\right)$ as the amalgamated product of two free groups such that $g$ is cyclically reduced of syllable length $\geq 2$ (see Proposition 4.4). Afterwards, we take an element $h \in \pi_{1}\left(\Sigma_{\rho}\right)$ which is not conjugate to $g$, and we prove that there exists a homomorphism $\varphi: F_{1} *_{C} F_{2} \rightarrow P_{1} *_{\bar{C}} P_{2}$ such that $P_{1} *_{\bar{C}} P_{2}$ is the amalgamated product of two finite $p$-groups along a cyclic subgroup $\bar{C}, \varphi(g)$ is cyclically reduced, $\operatorname{Syl}(\varphi(g))=\operatorname{Syl}(g) \geq 2$, and $\varphi(h) \nsim \varphi(g)$ (see Proposition 4.5). We conclude using the following.

Theorem 4.3 (Ivanova, [12]). Let $H=P_{1} *_{\bar{C}} P_{2}$ be the amalgamated product of two finite p-groups $P_{1}, P_{2}$ along a cyclic group $\bar{C}$. Then every cyclically reduced element of $H$ of syllable length $\geq 2$ is conjugacy p-distinguished.

Remark. The exact result which can be found in [12] is that: if the amalgamated product $P_{1} *_{\bar{C}} P_{2}$ of two finite $p$-groups is residually $p$, then any element of $P_{1} *_{\bar{C}} P_{2}$ of infinite order is conjugacy $p$-distinguished. The fact that $g$ is of infinite order if it is cyclically reduced and of syllable length $\geq 2$ is a classical fact that can be found for instance in [20]. The fact that $P_{1} *_{\bar{C}} P_{2}$ is residually $p$ if $\bar{C}$ is cyclic is proved 
in 9. Note that the amalgamated product of two finite $p$-groups is not residually $p$ in general (see [9]).

We now turn to the first step of our proof of Theorem 4.1.

Proposition 4.4. Let $g \in \pi_{1}\left(\Sigma_{\rho}\right) \backslash\{1\}$. Then there exists a decomposition $\pi_{1}\left(\Sigma_{\rho}\right)=$ $F_{1} *_{C} F_{2}$ of $\pi_{1}\left(\Sigma_{\rho}\right)$ as an amalgamated product such that $F_{1}=F\left(x_{1}, \ldots, x_{n}, y_{1}, \ldots, y_{n}\right)$ is a free group of rank $2 n, F_{2}=F\left(x_{1}^{\prime}, \ldots, x_{m}^{\prime}, y_{1}^{\prime}, \ldots, y_{m}^{\prime}\right)$ is a free group of rank $2 m, C$ is the cyclic subgroup generated by

$$
\gamma=\left[x_{1}, y_{1}\right] \cdots\left[x_{n}, y_{n}\right]=\left[x_{1}^{\prime}, y_{1}^{\prime}\right] \cdots\left[x_{m}^{\prime}, y_{m}^{\prime}\right]
$$

and the element $g \in F_{1} *_{C} F_{2}$ is cyclically reduced of syllable length $\geq 2$.

Proof. Let $a: \mathbb{S}^{1} \hookrightarrow \Sigma_{\rho}$ be a separating simple closed curve of $\Sigma_{\rho}$ (see Figure 4.1). The curve $a$ separates $\Sigma_{\rho}$ into two subsurfaces $\Sigma_{1}$ and $\Sigma_{2}$, each of them with a unique boundary component. Let $a_{1}: \mathbb{S}^{1} \hookrightarrow \partial \Sigma_{1}$ (resp. $\left.a_{2}: \mathbb{S}^{1} \hookrightarrow \partial \Sigma_{2}\right)$ be the boundary curve of $\Sigma_{1}$ (resp. of $\left.\Sigma_{2}\right)$. Then $\Sigma_{\rho}=\left(\Sigma_{1} \sqcup \Sigma_{2}\right) / \sim$, where $\sim$ is the equivalence relation which identifies $a_{1}(z)$ with $a_{2}(\bar{z})$ for all $z \in \mathbb{S}^{1}$. Set $B_{0}=a(1)$, let $\bar{a}:[0,1] \rightarrow \Sigma_{\rho}$ be the loop based at $B_{0}$ defined by $\bar{a}(t)=a\left(e^{2 i \pi t}\right)$, and let $\gamma \in \pi_{1}\left(\Sigma_{\rho}, B_{0}\right)$ be the class of $\bar{a}$. Then $\pi_{1}\left(\Sigma_{\rho}\right)$ has the amalgamated decomposition $\pi_{1}\left(\Sigma_{\rho}, B_{0}\right)=\pi_{1}\left(\Sigma_{1}, B_{0}\right) *_{C} \pi_{1}\left(\Sigma_{2}, B_{0}\right)$, where $C$ is the infinite cyclic subgroup generated by $\gamma$. Let $n$ (resp. $m$ ) be the genus of $\Sigma_{1}$ (resp. $\Sigma_{2}$ ). Then $\pi_{1}\left(\Sigma_{1}, B_{0}\right)=F_{1}=F\left(x_{1}, \ldots, x_{n}, y_{1}, \ldots, y_{n}\right)$ is a free group of rank $2 n, \pi_{1}\left(\Sigma_{2}, B_{0}\right)=$ $F_{2}=F\left(x_{1}^{\prime}, \ldots, x_{m}^{\prime}, y_{1}^{\prime}, \ldots, y_{m}^{\prime}\right)$ is a free group of rank $2 m$, and the generators can be chosen so that

$$
\gamma=\left[x_{1}, y_{1}\right] \cdots\left[x_{n}, y_{n}\right]=\left[x_{1}^{\prime}, y_{1}^{\prime}\right] \cdots\left[x_{m}^{\prime}, y_{m}^{\prime}\right] .
$$

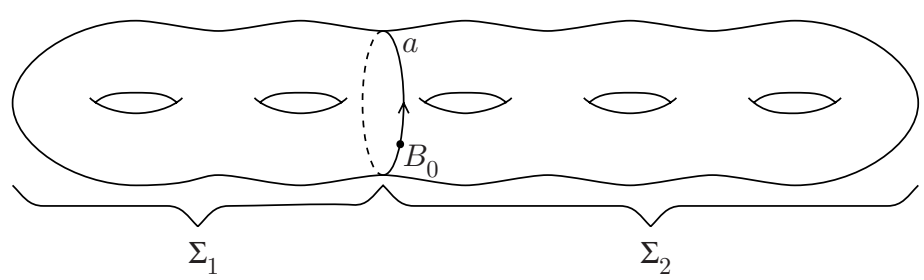

FiguRE 4.1. Amalgamated decomposition of $\pi_{1}\left(\Sigma_{\rho}\right)$.

Let $g \in \pi_{1}\left(\Sigma_{\rho}, B_{0}\right) \backslash\{1\}$. Let $\bar{c}_{g}:[0,1] \rightarrow \Sigma_{\rho}$ be a loop based at $B_{0}$ which represents $g$, and let $c_{g}: \mathbb{S}^{1} \rightarrow \Sigma_{\rho}$ be the closed curve defined by $c_{g}\left(e^{2 i \pi t}\right)=\bar{c}_{g}(t)$. It is easily shown that an element $h \in \pi_{1}\left(\Sigma_{\rho}, B_{0}\right)$ is conjugate to $g$ if and only if $c_{h}$ is homotopic to $c_{g}$ (here the homotopies are free with no fixed point).

Let $c: \mathbb{S}^{1} \rightarrow \Sigma_{\rho}$ be a closed curve homotopic to $c_{g}$ and which intersects $a$ transversely. Let $\bar{c}:[0,1] \rightarrow \Sigma_{\rho}$ be the loop defined by $\bar{c}(t)=c\left(e^{2 i \pi t}\right)$, and let $0 \leq t_{1}<t_{2}<\cdots<t_{l}<1$ such that $\bar{c}\left(t_{i}\right) \in a\left(\mathbb{S}^{1}\right)$ for all $1 \leq i \leq l$, and $\bar{c}(t) \notin a\left(\mathbb{S}^{1}\right)$ if $t \notin\left\{t_{1}, \ldots, t_{l}\right\}$. In other words, we have $c\left(\mathbb{S}^{1}\right) \cap a\left(\mathbb{S}^{1}\right)=\left\{\bar{c}\left(t_{1}\right), \ldots, \bar{c}\left(t_{l}\right)\right\}$, where the points are counted with multiplicity. For $1 \leq i \leq l$, let $s_{i} \in[0,1[$ such that $\bar{c}\left(t_{i}\right)=\bar{a}\left(s_{i}\right)$, and let

$$
h_{i}=\left[\left.\left.\bar{a}\right|_{\left[0, s_{i}\right]} \cdot \bar{c}\right|_{\left[t_{i}, t_{i+1}\right]} \cdot\left(\left.\bar{a}\right|_{\left[0, s_{i+1}\right]}\right)^{-1}\right] \in \pi_{1}\left(\Sigma_{\rho}, B_{0}\right)
$$


(here we assume $t_{l+1}=t_{1}, s_{l+1}=s_{1}$, and $\left.\left[t_{l}, t_{1}\right]=\left[t_{l}, 1\right] \cup\left[0, t_{1}\right]\right)$. We have either $\bar{c}\left(\left[t_{i}, t_{i+1}\right]\right) \subset \Sigma_{1}$ or $\bar{c}\left(\left[t_{i}, t_{i+1}\right]\right) \subset \Sigma_{2}$. Moreover, if $\bar{c}\left(\left[t_{i}, t_{i+1}\right]\right) \subset \Sigma_{1}$ (resp. $\left.\bar{c}\left(\left[t_{i}, t_{i+1}\right]\right) \subset \Sigma_{2}\right)$, then $h_{i} \in \pi_{1}\left(\Sigma_{1}, B_{0}\right)=F_{1}\left(\right.$ resp. $\left.h_{i} \in \pi_{1}\left(\Sigma_{2}, B_{0}\right)=F_{2}\right)$.

If $h_{i} \in\langle\gamma\rangle=C$, then there is a closed curve $c^{\prime}$ homotopic to $c$ such that $\bar{c}^{\prime}$ follows $\bar{c}$ between 0 and $t_{i}-\varepsilon, \bar{c}^{\prime}$ follows $\bar{c}$ between $t_{i+1}+\varepsilon$ and 1 , and $\bar{c}^{\prime}$ remains in the same component of $\Sigma_{\rho} \backslash a\left(\mathbb{S}^{1}\right)$ between $t_{i}-\varepsilon$ and $t_{i+1}+\varepsilon$. Hence, we can reduce the number $l$ in this manner. So, if $l$ is minimal, then

$$
h=h_{1} \cdot h_{2} \cdots h_{l}
$$

is a normal form. It is also cyclically reduced because $l$ is even (since $a$ is separating) and $h$ is conjugate to $g$. We set $l=I(a, g)$. By Proposition 4.2, this number is well-defined.

Now, our goal is to prove that we can choose $a$ so that $I(a, g) \neq 0$. We assume that $I(a, g)=0$ for every separating simple closed curve $a: \mathbb{S}^{1} \hookrightarrow \Sigma_{\rho}$, and we look for a contradiction.

We take $2 \rho-3$ separating simple closed curves $a_{1}, \ldots, a_{2 \rho-3}: \mathbb{S}^{1} \hookrightarrow \Sigma_{\rho}$ that are pairwise disjoint and such that the components of the natural compactification of $\Sigma_{\rho} \backslash\left(\bigcup_{i=1}^{2 \rho-3} a_{i}\left(\mathbb{S}^{1}\right)\right)$ are all pantalons (spheres with 3 holes) or tori with one hole (see Figure 4.2). Since $I\left(a_{i}, g\right)=0$ for all $1 \leq i \leq 2 \rho-3$, we can assume that $c$ is included in a component of $\Sigma_{\rho} \backslash\left(\bigcup_{i=1}^{2 \rho-3} a_{i}\left(\mathbb{S}^{1}\right)\right)$.

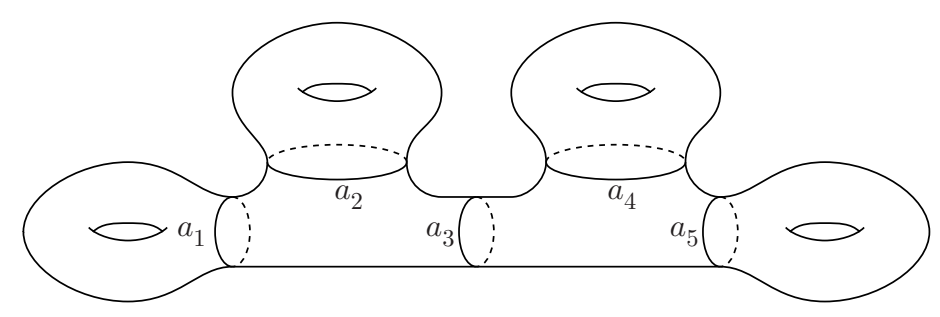

Figure 4.2. A decomposition of $\Sigma_{\rho}$ into pantalons and one-hole tori.

Let $S_{0}$ be a tubular neighborhood of $c$, and let $d_{1}, \ldots, d_{r}: \mathbb{S}^{1} \hookrightarrow \partial S_{0}$ be the boundary curves of $S_{0}$. Up to permutation, we can assume that $d_{i}$ bounds a disk $\mathbb{D}_{i}$ in $\Sigma_{\rho} \backslash S_{0}$ for all $k+1 \leq i \leq r$, and $d_{i}$ does not bound any disk for all $1 \leq i \leq k$. Set $S=S_{0} \cup\left(\bigcup_{i=k+1}^{r} \mathbb{D}_{i}\right)$. Then $S$ is an essential subsurface of $\Sigma_{\rho}$ (i.e. $\pi_{1}(S)$ embeds in $\pi_{1}\left(\Sigma_{\rho}\right)$ ) which can be assumed to be included in some component of $\Sigma_{\rho} \backslash\left(\bigcup_{i=1}^{2 \rho-3} a_{i}\left(\mathbb{S}^{1}\right)\right)$. In particular, $S$ is either an annulus, a pantalon, or a one-hole torus.

We denote by $I(x, y)$ the geometric intersection number of two simple closed curves $x, y$ (see [1]). Let $d: \mathbb{S}^{1} \hookrightarrow \Sigma_{\rho}$ be a boundary curve of $S$. Let $a: \mathbb{S}^{1} \hookrightarrow \Sigma_{\rho}$ be a separating simple closed curve of $\Sigma_{\rho}$ such that $I(a, d) \geq 2$ (the proof of the existence of such a curve is left to the reader). Let $a^{\prime}$ be an arc of $a$ whose extremities, $P$ and $Q$, belong to $S$, and let $x$ be a path in $S$ which joins $P$ to $Q$. We consider $a^{\prime} \cup x$ as a closed curve. Then one can prove (with some effort) that: if $a^{\prime} \cup x$ is homotopic to a constant curve, then $a^{\prime}$ is included in $S$. This fact and the equality $I(a, g)=0$ imply that $c$ is homotopic to a curve $c^{\prime}$ included in $S$ and such that $c^{\prime} \cap a=\emptyset$. So, $c^{\prime}$ is included in a connected component of $S \backslash a\left(\mathbb{S}^{1}\right)$. Such a component is either a disk or an annulus if $S$ is either a pantalon or a one-hole 
torus, and is a disk if $S$ is an annulus. We conclude that $c_{g}$ is homotopic to a constant curve, a contradiction.

The second step of our proof of Theorem 4.1 is given by the following.

Proposition 4.5. Let $\pi_{1}\left(\Sigma_{\rho}\right)=F_{1} *_{C} F_{2}$ be an amalgamated decomposition of $\pi_{1}\left(\Sigma_{\rho}\right)$ such that $F_{1}=F\left(x_{1}, \ldots, x_{n}, y_{1}, \ldots, y_{n}\right)$ is a free group of rank $2 n, F_{2}=$ $F\left(x_{1}^{\prime}, \ldots, x_{m}^{\prime}, y_{1}^{\prime}, \ldots, y_{m}^{\prime}\right)$ is a free group of rank $2 m$, and $C$ is the cyclic group generated by

$$
\gamma=\left[x_{1}, y_{1}\right] \cdots\left[x_{n}, y_{n}\right]=\left[x_{1}^{\prime}, y_{1}^{\prime}\right] \cdots\left[x_{m}^{\prime}, y_{m}^{\prime}\right]
$$

Let $g \in \pi_{1}\left(\Sigma_{\rho}\right)$ be a cyclically reduced element of syllable length $2 l \geq 2$, and let $h \in$ $\pi_{1}\left(\Sigma_{\rho}\right)$ be another element not conjugate to $g$. Then there exists a homomorphism $\varphi: F_{1} *_{C} F_{2} \rightarrow P_{1}{ }{ }_{\bar{C}} P_{2}$ such that $P_{1} *_{\bar{C}} P_{2}$ is the amalgamated product of two finite p-groups along a cyclic group $\bar{C}, \varphi(g)$ is cyclically reduced of syllable length equal to $2 l=\operatorname{Syl}(g)$, and $\varphi(h)$ is not conjugate to $\varphi(g)$.

The following Lemmas 4.6 to 4.9 are preliminaries to the proof of Proposition 4.5. Lemmas 4.6 and 4.9 are due to Stebe (see [21, Lemma 1 and Lemma 6). The proof of Lemma 4.8 is long and technical, so we put it separately in the last section.

Lemma 4.6 (Stebe, [21]). Let $F=F(X)$ be a free group, let $g \in F \backslash\{1\}$ be a non-trivial element, and let $q=p^{e}$ be a fixed power of $p$. Then there exists a homomorphism $\varphi: F \rightarrow P$ such that $P$ is a finite p-group and the order of $\varphi(g)$ is exactly $q$.

Lemma 4.7. Let $F=F\left(x_{1}, \ldots, x_{n}, y_{1}, \ldots, y_{n}\right)$ be a free group of rank $2 n$, let $\gamma=$ $\left[x_{1}, y_{1}\right] \cdots\left[x_{n}, y_{n}\right]$, and let $C$ be the cyclic subgroup generated by $\gamma$. Let $g \in F \backslash C$. Then there exists a homomorphism $\varphi: F \rightarrow P$ such that $P$ is a finite p-group and $\varphi(g) \notin \varphi(C)$.

Proof. Since $\gamma$ is primitive, the centralizer of $\gamma$ in $F$ is equal to $C$. Now, $g \notin C$, thus $[g, \gamma] \neq\{1\}$. The group $F$ is residually $p$, thus there exists a homomorphism $\varphi: F \rightarrow P$ such that $P$ is a finite $p$-group and $\varphi([g, \gamma])=[\varphi(g), \varphi(\gamma)] \neq 1$. It follows that $\varphi(g) \notin \varphi(C)=\langle\varphi(\gamma)\rangle$.

As pointed out before, the proof of the following is the object of Section 5.

Lemma 4.8. Let $F=F\left(x_{1}, \ldots, x_{n}, y_{1}, \ldots, y_{n}\right)$ be a free group of rank $2 n$, and let $\gamma=\left[x_{1}, y_{1}\right] \cdots\left[x_{n}, y_{n}\right]$. Let $g, h \in F$. If $\gamma^{a} g \neq h \gamma^{b}$ for all $a, b \in \mathbb{Z}$, then there exists a homomorphism $\varphi: F \rightarrow P$ such that $P$ is a finite p-group and $\varphi(\gamma)^{a} \varphi(g) \neq \varphi(h) \varphi(\gamma)^{b}$ for all $a, b \in \mathbb{Z}$.

Lemma 4.9 (Stebe, 21]). Let $P$ be a finite p-group. Let $\xi, \omega \in P$ such that $\left[\omega, \xi \omega \xi^{-1}\right] \neq 1$, and let $a, b \in \mathbb{Z}$. If $\omega^{a}=\xi \omega^{b} \xi^{-1}$, then $p$ divides $a$ and $b$.

Corollary 4.10. Let $P$ be a finite p-group. Let $e \geq 0$, let $\xi, \omega \in P$ such that $\left[\omega^{p^{r}}, \xi \omega^{p^{r}} \xi^{-1}\right] \neq 1$ for all $0 \leq r \leq e$, and let $a, b \in \mathbb{Z}$. If $\omega^{a}=\xi \omega^{b} \xi^{-1}$, then $p^{e+1}$ divides $a$ and $b$.

Proof. We show by induction on $r$ that $p^{r+1}$ divides $a$ and $b$ for all $0 \leq r \leq e$. The case $r=0$ is a direct consequence of Lemma 4.9. We assume $r \geq 1$. By induction, $p^{r}$ divides $a$ and $b$. We set $a=p^{r} a^{\prime}$ and $b=p^{r} b^{\prime}$. We have

$$
\left(\omega^{p^{r}}\right)^{a^{\prime}}=\xi\left(\omega^{p^{r}}\right)^{b^{\prime}} \xi^{-1}
$$

thus, by Lemma 4.9, $p$ divides $a^{\prime}$ and $b^{\prime}$, therefore $p^{r+1}$ divides $a$ and $b$. 
Proof of Proposition 4.5. We can assume that

$$
g=g_{1} \cdot g_{1}^{\prime} \cdots g_{l} \cdot g_{l}^{\prime}
$$

is a normal form of $g$, where $g_{i} \in F_{1} \backslash C$ and $g_{i}^{\prime} \in F_{2} \backslash C$ for all $1 \leq i \leq l$.

Step 1. We assume that $h \in F_{1}$.

By Lemma 4.7, there exists a homomorphism $\alpha_{1 i}: F_{1} \rightarrow A_{1 i}$ such that $A_{1 i}$ is a finite $p$-group and $\alpha_{1 i}\left(g_{i}\right) \notin \alpha_{1 i}(C)$ for all $1 \leq i \leq l$. Let $L_{1}=\bigcap_{i=1}^{l} \operatorname{Ker} \alpha_{1 i}$, let $B_{1}=F_{1} / L_{1}$, and let $\beta_{1}: F_{1} \rightarrow B_{1}$ be the quotient map. Then $B_{1}$ is a finite $p$ group (see Lemma 3.1) and $\beta_{1}\left(g_{i}\right) \notin \beta_{1}(C)$ for all $1 \leq i \leq l$. Similarly, there exists a homomorphism $\beta_{2}: F_{2} \rightarrow B_{2}$ such that $B_{2}$ is a finite $p$-group and $\beta_{2}\left(g_{i}^{\prime}\right) \notin \beta_{2}(C)$ for all $1 \leq i \leq l$. Let $q_{1}=p^{e_{1}}$ be the order of $\beta_{1}(\gamma)$, let $q_{2}=p^{e_{2}}$ be the order of $\beta_{2}(\gamma)$, and let $q=q_{1} q_{2}=p^{e_{1}+e_{2}}$. By Lemma 4.6, there exists a homomorphism $\psi_{j}: F_{j} \rightarrow Q_{j}$ such that $Q_{j}$ is a finite $p$-group and the order of $\psi_{j}(\gamma)$ is equal to $q$, for $j=1,2$. Let $K_{j}=\operatorname{Ker} \beta_{j} \cap \operatorname{Ker} \psi_{j}$, let $P_{j}=F_{j} / K_{j}$, and let $\varphi_{j}: F_{j} \rightarrow P_{j}$ be the quotient map, for $j=1,2$. Then $P_{1}$ is a finite $p$-group, $\varphi_{1}\left(g_{i}\right) \notin \varphi_{1}(C)$ for all $1 \leq i \leq l$, and the order of $\varphi_{1}(\gamma)$ is equal to $q$. Similarly, $P_{2}$ is a finite $p$-group, $\varphi_{2}\left(g_{i}^{\prime}\right) \notin \varphi_{2}(C)$ for all $1 \leq i \leq l$, and the order of $\varphi_{2}(\gamma)$ is equal to $q$. Let $H=P_{1} *_{\varphi_{1}(\gamma)=\varphi_{2}(\gamma)} P_{2}=P_{1} *_{\bar{C}} P_{2}$, where $\bar{C}$ is the cyclic group generated by $\varphi_{1}(\gamma)=\varphi_{2}(\gamma)$. Then the homomorphisms $\varphi_{1}, \varphi_{2}$ induce a homomorphism $\varphi: F_{1} *_{C} F_{2} \rightarrow P_{1} *_{\bar{C}} P_{2}$.

By construction,

$$
\varphi(g)=\varphi_{1}\left(g_{1}\right) \cdot \varphi_{2}\left(g_{1}^{\prime}\right) \cdots \varphi_{1}\left(g_{l}\right) \cdot \varphi_{2}\left(g_{l}^{\prime}\right)
$$

is a normal form which is cyclically reduced. Furthermore, we have $\varphi(h) \in P_{1}$, thus, by Proposition 4.2, $\varphi(h)$ is not conjugate to $\varphi(g)$.

Conclusion of Step 1. We can assume that $h$ is not conjugate to an element of $F_{1}$ and is not conjugate to an element of $F_{2}$. So, we can assume that $h$ is cyclically reduced of syllable length $2 k \geq 2$ and has a normal form

$$
h=h_{1} \cdot h_{1}^{\prime} \cdots h_{k} \cdot h_{k}^{\prime}
$$

with $h_{j} \in F_{1} \backslash C$ and $h_{j}^{\prime} \in F_{2} \backslash C$ for all $1 \leq j \leq k$.

Step 2. We assume that $k \neq l$.

It is easily proved using the same arguments as in Step 1 that there exist homomorphisms $\varphi_{1}: F_{1} \rightarrow P_{1}, \varphi_{2}: F_{2} \rightarrow P_{2}$ such that $P_{1}$ and $P_{2}$ are finite $p$-groups, $\varphi_{1}\left(g_{i}\right), \varphi_{1}\left(h_{j}\right) \notin \varphi_{1}(C)$ for all $1 \leq i \leq l$ and all $1 \leq j \leq k, \varphi_{2}\left(g_{i}^{\prime}\right), \varphi_{2}\left(h_{j}^{\prime}\right) \notin \varphi_{2}(C)$ for all $1 \leq i \leq l$ and all $1 \leq j \leq k$, and the order of $\varphi_{1}(\gamma)$ is equal to the order of $\varphi_{2}(\gamma)$. Let $H=P_{1} *_{\varphi_{1}(\gamma)=\varphi_{2}(\gamma)} P_{2}=P_{1} *_{\bar{C}} P_{2}$, where $\bar{C}$ is the cyclic group generated by $\varphi_{1}(\gamma)=\varphi_{2}(\gamma)$. Then $\varphi_{1}$ and $\varphi_{2}$ induce a homomorphism $\varphi: F_{1} *_{C} F_{2} \rightarrow P_{1} *_{\bar{C}} P_{2}$.

By construction

$$
\varphi(g)=\varphi_{1}\left(g_{1}\right) \cdot \varphi_{2}\left(g_{1}^{\prime}\right) \cdots \varphi_{1}\left(g_{l}\right) \cdot \varphi_{2}\left(g_{l}^{\prime}\right) \quad \text { and } \quad \varphi(h)=\varphi_{1}\left(h_{1}\right) \cdot \varphi_{2}\left(h_{1}^{\prime}\right) \cdots \varphi_{1}\left(h_{k}\right) \cdot \varphi_{2}\left(h_{k}^{\prime}\right)
$$

are cyclically reduced normal forms. By Proposition 4.2, we conclude that $\varphi(g) \nsim$ $\varphi(h)$. 
Conclusion of Step 2. We can assume $k=l$.

For the next step we need the following.

Claim 1. Let $f \in F_{1} \backslash C$ and $n \in \mathbb{Z} \backslash\{0\}$. Then $\left[\gamma^{n}, f \gamma^{n} f^{-1}\right] \neq 1$.

Proof of Claim 1. Suppose that $\left[\gamma^{n}, f \gamma^{n} f^{-1}\right]=1$. Let $H$ be the subgroup of $F_{1}$ generated by $\{\gamma, f\}$. Either $H$ is free of rank 2 freely generated by $\{\gamma, f\}$, or $H$ is cyclic. Since $\left[\gamma^{n}, f \gamma^{n} f^{-1}\right]=1$, the group $H$ is not freely generated by $\{\gamma, f\}$, thus $H$ is a cyclic group. The element $\gamma$ is primitive, thus $H$ is equal to $\langle\gamma\rangle=C$, therefore $f \in C$, a contradiction.

Step 3. We construct a homomorphism $\varphi: F_{1} *_{C} F_{2} \rightarrow P_{1} *_{\bar{C}} P_{2}$, where $P_{1}, P_{2}$ are finite $p$-groups, $\bar{C}$ is the cyclic group generated by $\varphi(\gamma)$, and $\operatorname{Syl}(\varphi(g))=$ $\operatorname{Syl}(\varphi(h))=2 l$.

For $1 \leq t \leq l$, we set

$$
h^{(t)}=h_{t+1} \cdot h_{t+1}^{\prime} \cdots h_{t+l} \cdot h_{t+l}^{\prime},
$$

where the indices are considered $\bmod l$. By Proposition 4.2, the fact that $g$ is not conjugate to $h$ is equivalent to

$$
\gamma^{a} h^{(t)} \gamma^{-a} \neq g \text { for all } a \in \mathbb{Z} \text { and all } 1 \leq t \leq l .
$$

It is easily proved using the same arguments as in Step 1 that there exist homomorphisms $\alpha_{1}: F_{1} \rightarrow A_{1}, \alpha_{2}: F_{2} \rightarrow A_{2}$ such that $A_{1}, A_{2}$ are finite $p$-groups, $\alpha_{1}\left(g_{i}\right), \alpha_{1}\left(h_{i}\right) \notin \alpha_{1}(C)$ for all $1 \leq i \leq l$, and $\alpha_{2}\left(g_{i}^{\prime}\right), \alpha_{2}\left(h_{i}^{\prime}\right) \notin \alpha_{2}(C)$ for all $1 \leq i \leq l$.

Now, for a given $1 \leq t \leq l$, we construct homomorphisms $\beta_{1}^{(t)}: F_{1} \rightarrow B_{1}^{(t)}$, $\beta_{2}^{(t)}: F_{2} \rightarrow B_{2}^{(t)}$, where $B_{1}^{(t)}, B_{2}^{(t)}$ are finite $p$-groups.

Case 1. There exists $1 \leq i \leq l$ such that $\gamma^{a} h_{t+i} \neq g_{i} \gamma^{b}$ for all $a, b \in \mathbb{Z}$.

By Lemma 4.8, there exists a homomorphism $\beta_{1}^{(t)}: F_{1} \rightarrow B_{1}^{(t)}$ such that $B_{1}^{(t)}$ is a finite $p$-group and $\beta_{1}^{(t)}\left(\gamma^{a} h_{t+i}\right) \neq \beta_{1}^{(t)}\left(g_{i} \gamma^{b}\right)$ for all $a, b \in \mathbb{Z}$. On the other hand, we set $B_{2}^{(t)}=\{1\}$, and $\beta_{2}^{(t)}: F_{2} \rightarrow B_{2}^{(t)}$ is the trivial map.

Case 2. Not in Case 1, and there exists $1 \leq i \leq l$ such that $\gamma^{a} h_{t+i}^{\prime} \neq g_{i}^{\prime} \gamma^{b}$ for all $a, b \in \mathbb{Z}$.

By Lemma 4.8, there exists a homomorphism $\beta_{2}^{(t)}: F_{2} \rightarrow B_{2}^{(t)}$ such that $B_{2}^{(t)}$ is a finite $p$-group and $\beta_{2}^{(t)}\left(\gamma^{a} h_{t+i}^{\prime}\right) \neq \beta_{2}^{(t)}\left(g_{i}^{\prime} \gamma^{b}\right)$ for all $a, b \in \mathbb{Z}$. On the other hand, we set $B_{1}^{(t)}=\{1\}$, and $\beta_{1}^{(t)}: F_{1} \rightarrow B_{1}^{(t)}$ is the trivial map.

Case 3. For all $1 \leq i \leq l$ there exist $a_{i}, a_{i}^{\prime}, b_{i}, b_{i}^{\prime} \in \mathbb{Z}$ such that $\gamma^{a_{i}} h_{t+i}=g_{i} \gamma^{b_{i}}$ and $\gamma^{a_{i}^{\prime}} h_{t+i}^{\prime}=g_{i}^{\prime} \gamma^{b_{i}^{\prime}}$.

Set $c_{i}=b_{i}-a_{i}^{\prime}$ for $1 \leq i \leq l, c_{i}^{\prime}=b_{i}^{\prime}-a_{i+1}$ for $1 \leq i \leq l-1$, and $c_{l}^{\prime}=b_{l}^{\prime}-a_{1}$. Then

$$
\gamma^{a_{1}} h^{(t)} \gamma^{-a_{1}}=g_{1} \gamma^{c_{1}} g_{1}^{\prime} \gamma^{c_{1}^{\prime}} \cdots g_{l} \gamma^{c_{l}} g_{l}^{\prime} \gamma^{c_{l}^{\prime}}
$$

Moreover, since $h$ is not conjugate to $g$, not all the $c_{i}$ 's or the $c_{i}^{\prime}$ 's are zero. Let

$$
u=\operatorname{gcd}\left(c_{1}, \ldots, c_{l}, c_{1}^{\prime}, \ldots, c_{l}^{\prime}\right),
$$

and write $u=p^{e} v$, where $p$ does not divide $v$. Since $F_{1}$ is residually $p$, by Claim 1 there exists a homomorphism $\beta_{1}^{(t)}: F_{1} \rightarrow B_{1}^{(t)}$ such that $B_{1}^{(t)}$ is a finite $p$-group and

$$
\beta_{1}^{(t)}\left(\left[\gamma^{p^{r}}, g_{i}^{-1} \gamma^{p^{r}} g_{i}\right]\right) \neq 1
$$


for all $1 \leq i \leq l$ and all $0 \leq r \leq e$. Similarly, there exists a homomorphism $\beta_{2}^{(t)}: F_{2} \rightarrow B_{2}^{(t)}$ such that $B_{2}^{(t)}$ is a finite $p$-group and

$$
\beta_{2}^{(t)}\left(\left[\gamma^{p^{r}}, g_{i}^{\prime-1} \gamma^{p^{r}} g_{i}^{\prime}\right]\right) \neq 1
$$

for all $1 \leq i \leq l$ and all $0 \leq r \leq e$.

Now, let $L_{j}=\operatorname{Ker} \alpha_{j} \cap\left(\bigcap_{t=1}^{l} \operatorname{Ker} \beta_{j}^{(t)}\right)$, let $N_{j}=F_{j} / L_{j}$, and let $\psi_{j}: F_{j} \rightarrow N_{j}$ be the quotient map, for $j=1,2$. Let $q_{1}=p^{e_{1}}$ be the order of $\psi_{1}(\gamma)$, let $q_{2}=p^{e_{2}}$ be the order of $\psi_{2}(\gamma)$, and let $q=q_{1} q_{2}=p^{e_{1}+e_{2}}$. By Lemma 4.6, there exist homomorphisms $\psi_{1}^{\prime}: F_{1} \rightarrow N_{1}^{\prime}, \psi_{2}^{\prime}: F_{2} \rightarrow N_{2}^{\prime}$ such that $N_{1}^{\prime}$ and $N_{2}^{\prime}$ are finite $p$-groups, and the order of $\psi_{j}^{\prime}(\gamma)$ is equal to $q$ for $j=1,2$.

Let $K_{j}=\operatorname{Ker} \psi_{j} \cap \operatorname{Ker} \psi_{j}^{\prime}$, let $P_{j}=F_{j} / K_{j}$, and let $\varphi_{j}: F_{j} \rightarrow P_{j}$ be the quotient map, for $j=1,2$. The groups $P_{1}, P_{2}$ are finite $p$-groups, and the orders of $\varphi_{1}(\gamma)$ and $\varphi_{2}(\gamma)$ are both equal to $q=p^{e_{1}+e_{2}}$. Let $H=P_{1} *_{\varphi_{1}(\gamma)=\varphi_{2}(\gamma)} P_{2}=P_{1} *_{\bar{C}} P_{2}$, where $\bar{C}$ is the cyclic group generated by $\varphi_{1}(\gamma)=\varphi_{2}(\gamma)$. Then $\varphi_{1}$ and $\varphi_{2}$ induce a homomorphism $\varphi: F_{1} *_{C} F_{2} \rightarrow P_{1} *_{\bar{C}} P_{2}$.

Step 4. We prove that $\varphi(h) \nsim \varphi(g)$.

By construction,

$\varphi(g)=\varphi_{1}\left(g_{1}\right) \cdot \varphi_{2}\left(g_{1}^{\prime}\right) \cdots \varphi_{1}\left(g_{l}\right) \cdot \varphi_{2}\left(g_{l}^{\prime}\right) \quad$ and $\quad \varphi(h)=\varphi_{1}\left(h_{1}\right) \cdot \varphi_{2}\left(h_{1}^{\prime}\right) \cdots \varphi_{1}\left(h_{l}\right) \cdot \varphi_{2}\left(h_{l}^{\prime}\right)$ are cyclically reduced normal forms. So, by Proposition 4.2, it suffices to show that

$$
\varphi(g) \neq \varphi\left(\gamma^{a} h^{(t)} \gamma^{-a}\right)
$$

for all $a \in \mathbb{Z}$ and all $1 \leq t \leq l$.

Case 1. There exists $1 \leq i \leq l$ such that $\gamma^{a} h_{t+i} \neq g_{i} \gamma^{b}$ for all $a, b \in \mathbb{Z}$.

By construction of $\beta_{1}^{(t)}$, we have

$$
\varphi_{1}\left(\gamma^{a} h_{t+i}\right) \neq \varphi_{1}\left(g_{i} \gamma^{b}\right)
$$

for all $a, b \in \mathbb{Z}$. By Proposition 4.2, it follows that $\varphi\left(\gamma^{a} h^{(t)} \gamma^{-a}\right) \neq \varphi(g)$ for all $a \in \mathbb{Z}$.

Case 2. Not in Case 1, and there exists $1 \leq i \leq l$ such that $\gamma^{a} h_{t+i}^{\prime} \neq g_{i}^{\prime} \gamma^{b}$ for all $a, b \in \mathbb{Z}$.

Then it is easily proved using the same arguments as in Case 1 that $\varphi\left(\gamma^{a} h^{(t)} \gamma^{-a}\right)$ $\neq \varphi(g)$ for all $a \in \mathbb{Z}$.

Case 3. For all $1 \leq i \leq l$ there exist $a_{i}, a_{i}^{\prime}, b_{i}, b_{i}^{\prime} \in \mathbb{Z}$ such that $\gamma^{a_{i}} h_{t+i}=g_{i} \gamma^{b_{i}}$ and $\gamma^{a_{i}^{\prime}} h_{t+i}^{\prime}=g_{i}^{\prime} \gamma^{b_{i}^{\prime}}$.

Following the construction of the homomorphisms $\beta_{1}^{(t)}$ and $\beta_{2}^{(t)}$ we set

$$
\gamma^{a_{1}} h^{(t)} \gamma^{-a_{1}}=g_{1} \gamma^{c_{1}} g_{1}^{\prime} \gamma^{c_{1}^{\prime}} \cdots g_{l} \gamma^{c_{l}} g_{l}^{\prime} \gamma^{c_{l}^{\prime}}
$$

and $u=p^{e} v=\operatorname{gcd}\left(c_{1}, \ldots, c_{l}, c_{1}^{\prime}, \ldots, c_{l}^{\prime}\right)$, where $p$ does not divide $v$. Suppose there exists $d \in \mathbb{Z}$ such that $\varphi\left(\gamma^{d} h^{(t)} \gamma^{-d}\right)=\varphi(g)$. By Proposition 4.2, there exist $d_{1}, \ldots, d_{l}, d_{1}^{\prime}, \ldots, d_{l}^{\prime} \in \mathbb{Z}$ such that

$$
\varphi\left(\gamma^{d_{i-1}^{\prime}} g_{i} \gamma^{c_{i}}\right)=\varphi\left(g_{i} \gamma^{d_{i}}\right) \text { and } \varphi\left(\gamma^{d_{i}} g_{i}^{\prime} \gamma^{c_{i}^{\prime}}\right)=\varphi\left(g_{i}^{\prime} \gamma^{d_{i}^{\prime}}\right)
$$

for all $1 \leq i \leq l$, where $d_{0}^{\prime}=d_{l}^{\prime}$. Let $1 \leq i \leq l$. The above equality implies that

$$
\varphi_{1}\left(g_{i}^{-1} \gamma^{d_{i-1}^{\prime}} g_{i}\right)=\varphi_{1}\left(\gamma^{d_{i}-c_{i}}\right) .
$$


Moreover, by construction of $\beta_{1}^{(t)}, \varphi_{1}\left(\left[\gamma^{p^{r}}, g_{i}^{-1} \gamma^{p^{r}} g_{i}\right]\right) \neq 1$ for all $0 \leq r \leq e$; thus, by Corollary 4.10, $p^{e+1}$ divides $d_{i-1}^{\prime}$ and $d_{i}-c_{i}$. Similarly, $p^{e+1}$ divides $d_{i}$ and $d_{i}^{\prime}-c_{i}^{\prime}$ for all $1 \leq i \leq l$. It follows that $p^{e+1}$ divides all the $c_{i}$ 's and all the $c_{i}^{\prime}$ 's, a contradiction. So, $\varphi\left(\gamma^{d} h^{(t)} \gamma^{-d}\right) \neq \varphi(g)$ for all $d \in \mathbb{Z}$.

Proof of Theorem 4.1. Let $g, h \in \pi_{1}\left(\Sigma_{\rho}\right)$ such that $g \nsim h$. We can assume $g \neq 1$. By Proposition 4.4, the group $\pi_{1}\left(\Sigma_{\rho}\right)$ has an amalgamated decomposition $\pi_{1}\left(\Sigma_{\rho}\right)=$ $F_{1} *_{C} F_{2}$, where $F_{1}=F\left(x_{1}, \ldots, x_{n}, y_{1}, \ldots, y_{n}\right)$ is a free group of rank $2 n, F_{2}=$ $F\left(x_{1}^{\prime}, \ldots, x_{m}^{\prime}, y_{1}^{\prime}, \ldots, y_{m}^{\prime}\right)$ is a free group of rank $2 m$, and $C$ is the cyclic group generated by

$$
\gamma=\left[x_{1}, y_{1}\right] \cdots\left[x_{n}, y_{n}\right]=\left[x_{1}^{\prime}, y_{1}^{\prime}\right] \cdots\left[x_{m}^{\prime}, y_{m}^{\prime}\right],
$$

and such that $g$ is cyclically reduced of syllable length $\geq 2$. By Proposition 4.5, there exists a homomorphism $\alpha: F_{1} *_{C} F_{2} \rightarrow P_{1} *_{\bar{C}} P_{2}$ such that $P_{1}, P_{2}$ are finite $p$ groups, $\bar{C}$ is the cyclic group generated by $\alpha(\gamma), \alpha(g)$ is cyclically reduced of syllable length $\geq 2$, and $\alpha(g) \nsim \alpha(h)$. By Theorem 4.3, there exists a homomorphism $\beta: P_{1} *_{\bar{C}} P_{2} \rightarrow P$ such that $P$ is a finite $p$-group and $\beta(\alpha(g)) \nsim \beta(\alpha(h))$. Let $\varphi=\beta \circ \alpha: \pi_{1}\left(\Sigma_{\rho}\right) \rightarrow P$. Then $\varphi(g) \nsim \varphi(h)$.

\section{A TECHNiCAL LEMMA}

Let $F=F\left(x_{1}, \ldots, x_{n}, y_{1}, \ldots, y_{n}\right)$ a free group of rank $2 n$, and let $\gamma=\left[x_{1}, y_{1}\right] \ldots$ $\left[x_{n}, y_{n}\right]$. In this section we prove the following.

Lemma 4.8. Let $g, h \in F$. If $\gamma^{a} g \neq h \gamma^{b}$ for all $a, b \in \mathbb{Z}$, then there exists a homomorphism $\varphi: F \rightarrow P$ such that $P$ is a finite $p$-group and $\varphi\left(\gamma^{a} g\right) \neq \varphi\left(h \gamma^{b}\right)$ for all $a, b \in \mathbb{Z}$.

The following Lemmas 5.1 and 5.2 are preliminaries to the proof of Lemma 4.8.

Lemma 5.1. Let $g \in F$. Then there exist a homomorphism $\varphi: F \rightarrow \mathbb{Z} / p^{2} \mathbb{Z}$ and a free generating set $Z$ for $\operatorname{Ker} \varphi$, such that $\gamma \in Z$, and $g \gamma g^{-1}$ is of the form $g \gamma g^{-1}=w z w^{-1}$, with $w \in \operatorname{Ker} \varphi$ and $z \in Z$.

Proof. Let $\Sigma$ be a surface of genus $n$ with one hole, and let $c: \mathbb{S}^{1} \hookrightarrow \partial \Sigma$ be the boundary curve of $\Sigma$. Let $B_{0}=c(1)$. Then we identify $F$ with $\pi_{1}\left(\Sigma, B_{0}\right)$ and $\gamma$ with the homotopy class of the loop $\bar{c}:[0,1] \rightarrow \Sigma, t \mapsto c\left(e^{2 i \pi t}\right)$.

Let $\tilde{\Sigma}$ be the surface of genus $p^{2}(n-1)+1$ with $p^{2}$ holes which we represent as follows.

Choose a small $\varepsilon>0$. For $1 \leq k \leq n-1$ and $0 \leq l \leq p^{2}-1$ we set

$$
O_{k l}=k e^{2 i \pi l / p^{2}}, \quad V_{k l}=(k-1+\varepsilon) e^{2 i \pi l / p^{2}}, \quad U_{k l}=(k-\varepsilon) e^{2 i \pi l / p^{2}} .
$$

These are complex numbers. We denote by $\mathcal{C}_{k l}$ the circle of radius $\varepsilon$ centered at $O_{k l}$, and by $\mathcal{D}_{k l}$ the interval $\mathcal{D}_{k l}=\left[V_{k l}, U_{k l}\right]$, for $1 \leq k \leq n-1$ and $0 \leq l \leq p^{2}-1$. We denote by $\mathcal{C}_{0}$ the circle centered at 0 of radius $\varepsilon$. We consider the connected graph

$$
\Gamma=\mathcal{C}_{0} \cup\left(\bigcup_{k, l} \mathcal{C}_{k l}\right) \cup\left(\bigcup_{k, l} \mathcal{D}_{k l}\right)
$$

embedded in $\mathbb{C}$ (see Figure 5.1).

We embed $\mathbb{C}$ in $\mathbb{R}^{3}=\mathbb{C} \times \mathbb{R}$ by $\xi \mapsto(\xi, 0)$, and we denote by $\tilde{\Sigma}_{0}$ the boundary of a regular neighborhood of $\Gamma$ in $\mathbb{R}^{3}$. Then $\tilde{\Sigma}_{0}$ is a closed oriented surface of genus $p^{2}(n-1)+1$. Let $H_{l}$ be the plane orthogonal to $\mathbb{R} e^{2 i \pi l / p^{2}}$ and which contains 


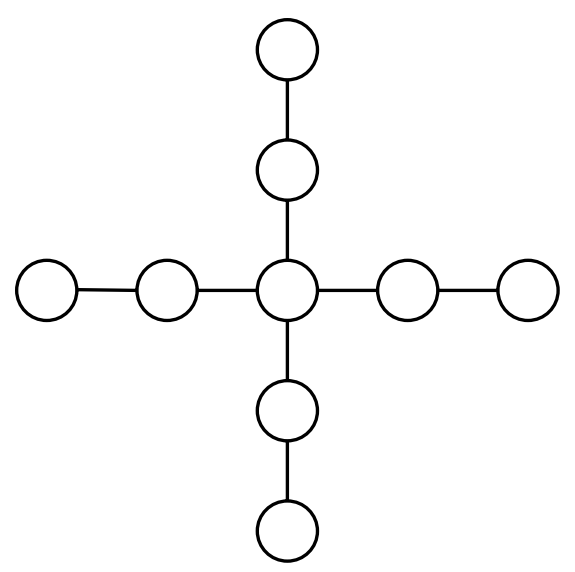

Figure 5.1. The graph $\Gamma$ for $n=3$ and $p=2$.

$\left(n-1+\varepsilon-\varepsilon^{2}\right) e^{2 i \pi l / p^{2}}$. It is easily seen that this construction can be made so that $H_{l} \cap \tilde{\Sigma}_{0}$ is a circle, $\tilde{c}_{l}$, which bounds a disk $\mathbb{D}_{l}$ embedded in $\tilde{\Sigma}_{0}$. We set $\tilde{\Sigma}=\tilde{\Sigma}_{0} \backslash\left(\bigcup_{l=0}^{p^{2}-1} \mathbb{D}_{l}\right)$. Then $\tilde{\Sigma}$ is an oriented surface of genus $p^{2}(n-1)+1$ with $p^{2}$ holes, and its boundary curves are $\tilde{c}_{0}, \tilde{c}_{1}, \ldots, \tilde{c}_{p^{2}-1}$.

Let $D=\{0\} \times \mathbb{R}$ be the central vertical line, and let $\theta: \mathbb{R}^{3} \rightarrow \mathbb{R}^{3}$ be the rotation of angle $2 \pi / p^{2}$ around $D$. Clearly, we can assume that $\theta(\tilde{\Sigma})=\tilde{\Sigma}$, and $\theta \circ \tilde{c}_{l}=\tilde{c}_{l+1}$ for all $0 \leq l \leq p^{2}-1$ (where the indices are considered $\left.\bmod p^{2}\right)$. Set $G=\langle\theta\rangle \simeq \mathbb{Z} / p^{2} \mathbb{Z}$. By the above, $G$ acts freely on $\tilde{\Sigma}$ and $\tilde{\Sigma} / G$ is a one-hole surface of genus $n$ which can be identified with $\Sigma$. Set $\tilde{B}_{l}=\tilde{c}_{l}(1)$ for all $0 \leq l \leq p^{2}-1$. So, we have a regular covering $\pi: \tilde{\Sigma} \rightarrow \Sigma$ (see Figure 5.2) which gives rise to an exact sequence

$$
1 \rightarrow \pi_{1}\left(\tilde{\Sigma}, \tilde{B}_{0}\right) \longrightarrow \pi_{1}\left(\Sigma, B_{0}\right) \longrightarrow G \simeq \mathbb{Z} / p^{2} \mathbb{Z} \rightarrow 1
$$

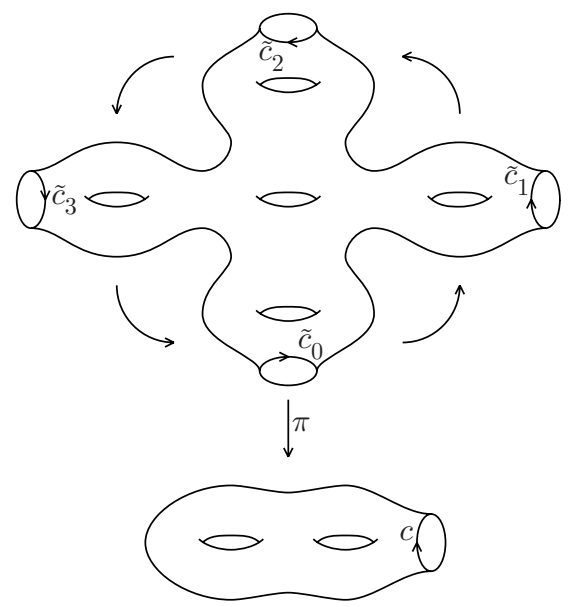

Figure 5.2. The regular covering $\tilde{\Sigma} \rightarrow \Sigma$ (for $n=2$ and $p=2$ ). 
For $0 \leq l \leq p^{2}-1$, we denote by $\hat{c}_{l}:[0,1] \rightarrow \tilde{\Sigma}$ the loop based at $\tilde{B}_{l}$ defined by $\hat{c}_{l}(t)=\tilde{c}_{l}\left(e^{2 i \pi t}\right)$, we choose a path $\hat{d}_{l}:[0,1] \rightarrow \tilde{\Sigma}$ from $\tilde{B}_{0}$ to $\tilde{B}_{l}$, and we set $z_{l}=\left[\hat{d}_{l} \hat{c}_{l} \hat{d}_{l}^{-1}\right] \in \pi_{1}\left(\tilde{\Sigma}, \tilde{B}_{0}\right)$. For a good choice of the $\hat{d}_{l}$ 's, the set $\left\{z_{0}, z_{1}, \ldots, z_{p^{2}-1}\right\}$ can be completed into a generating system

$$
\hat{Z}=\left\{u_{1}, \ldots, u_{p^{2}(n-1)+1}, v_{1}, \ldots, v_{p^{2}(n-1)+1}, z_{0}, z_{1}, \ldots, z_{p^{2}-1}\right\}
$$

for $\pi_{1}\left(\tilde{\Sigma}, \tilde{B}_{0}\right)$ so that $\pi_{1}\left(\tilde{\Sigma}, \tilde{B}_{0}\right)$ has a presentation with generating set $\hat{Z}$ and unique relation

$$
\left[u_{1}, v_{1}\right] \cdots\left[u_{p^{2}(n-1)+1}, v_{p^{2}(n-1)+1}\right]=z_{0} z_{1} \cdots z_{p^{2}-1} .
$$

In particular, for a chosen $k_{0} \in\left\{0,1, \ldots, p^{2}-1\right\}, \pi_{1}\left(\tilde{\Sigma}, \tilde{B}_{0}\right)$ is the free group freely generated by $Z=\hat{Z} \backslash\left\{z_{k_{0}}\right\}$. Also, the path $\hat{d}_{0}$ can and will be assumed to be the constant path.

Let $\omega:[0,1] \rightarrow \Sigma$ be a loop based at $B_{0}$ which represents $g$, let $\hat{\omega}:[0,1] \rightarrow \tilde{\Sigma}$ be the lift of $\omega$ such that $\hat{\omega}(0)=\tilde{B}_{0}$, and let $l_{0} \in\left\{0,1, \ldots, p^{2}-1\right\}$ such that $\hat{\omega}(1)=\tilde{B}_{l_{0}}$. Since $p^{2} \geq 3$, we can choose $k_{0}$ such that $k_{0} \neq 0$ and $k_{0} \neq l_{0}$. Then $\gamma=[c]=\left[\hat{c}_{0}\right]=z_{0} \in Z$ and

$$
g \gamma g^{-1}=\left[\hat{\omega} \hat{c}_{l_{0}} \hat{\omega}^{-1}\right]=w z_{l_{0}} w^{-1},
$$

where $w=\left[\hat{\omega} \hat{d}_{l_{0}}^{-1}\right] \in \pi_{1}\left(\tilde{\Sigma}, \tilde{B}_{0}\right)$.

Remark. In the above proof we only need the condition $p^{2} \geq 3$, so we can replace $p^{2}$ by $p$ if $p \neq 2$.

Lemma 5.2. Let $g, h \in F$. If $\gamma^{a} g \gamma^{b} g^{-1} \neq h$ for all $a, b \in \mathbb{Z}$, then there exists a homomorphism $\varphi: F \rightarrow P$ such that $P$ is a finite $p$-group and $\varphi\left(\gamma^{a} g \gamma^{b} g^{-1}\right) \neq \varphi(h)$ for all $a, b \in \mathbb{Z}$.

Proof. By Lemma 5.1, there exist a homomorphism $\pi: F \rightarrow \mathbb{Z} / p^{2} \mathbb{Z}$ and a free generating set $Z$ for $\operatorname{Ker} \pi$ such that $\gamma \in Z$ and $g \gamma g^{-1}$ is of the form $u z_{0} u^{-1}$, where $u \in \operatorname{Ker} \pi$ and $z_{0} \in Z$.

Suppose $\pi(h) \neq 1$. Set $P=\mathbb{Z} / p^{2} \mathbb{Z}$ and $\varphi=\pi: F \rightarrow P$. Then $\varphi\left(\gamma^{a} g \gamma^{b} g^{-1}\right)=$ $1 \neq \varphi(h)$ for all $a, b \in \mathbb{Z}$. So, we can assume that $h \in \operatorname{Ker} \pi$.

Let $u=\omega_{1}^{c_{1}} \omega_{2}^{c_{2}} \cdots \omega_{l}^{c_{l}}$ be the normal form of $u$ with respect to $Z$, where $c_{i} \in$ $\mathbb{Z} \backslash\{0\}, \omega_{i} \in Z$, and $\omega_{i} \neq \omega_{i+1}$ for all $i$. For convenience, we assume that $\omega_{1}=\gamma$ and that $c_{1}$ may be equal to 0 . Moreover, we can also assume that $\omega_{l} \neq z_{0}$. We set $v=\omega_{2}^{c_{2}} \cdots \omega_{l}^{c_{l}}$. For $a, b \in \mathbb{Z}$, the normal form of $\gamma^{a+c_{1}} v z_{0}^{b} v^{-1}$ is

$$
\begin{array}{ll}
\gamma^{a+c_{1}} & \text { if } b=0, \\
\gamma^{a+b+c_{1}} & \text { if } v=1 \text { and } z_{0}=\gamma, \\
\gamma^{a+c_{1}} \omega_{2}^{c_{2}} \cdots \omega_{l}^{c_{l}} z_{0}^{b} \omega_{l}^{-c_{l}} \cdots \omega_{2}^{-c_{2}} & \text { otherwise. }
\end{array}
$$

Let $h \gamma^{c_{1}}=z_{1}^{d_{1}} \cdots z_{k}^{d_{k}}$ be the normal form of $h \gamma^{c_{1}}$ with respect to $Z$. Let $q=p^{e}$ be a power of $p$ strictly greater than $2\left|c_{i}\right|$ and than $2\left|d_{j}\right|$ for all $2 \leq i \leq l$ and all $1 \leq j \leq k$.

Let

$$
\left.G=\langle Z| z^{q}=1 \text { for } z \in Z\right\rangle .
$$

The group $G$ is the free product of $|Z|$ copies of $\mathbb{Z} / q \mathbb{Z}$, and there is a natural homomorphism $\alpha$ : $\operatorname{Ker} \pi=F(Z) \rightarrow G$ which sends $z$ to $z$ for all $z \in Z$. Let

$$
U=\left\{\gamma^{a+c_{1}} v z_{0}^{b} v^{-1} ; a, b \in \mathbb{Z}\right\} .
$$


Note that the condition $\gamma^{a} g \gamma^{b} g^{-1} \neq h$ for all $a, b \in \mathbb{Z}$ is equivalent to $h \gamma^{c_{1}} \notin U$. Now, observe that $\alpha\left(h \gamma^{c_{1}}\right)=z_{1}^{b_{1}} \cdots z_{k}^{b_{k}}$ is the normal form of $\alpha\left(h \gamma^{c_{1}}\right)$ which, by construction, does not coincide with the normal form of any element of $\alpha(U)$, thus $\alpha\left(h \gamma^{c_{1}}\right) \notin \alpha(U)$.

The set $\alpha(U)$ is finite and the group $G$ is residually $p$, thus there exists a homomorphism $\beta: G \rightarrow B$ such that $B$ is a finite $p$-group and $(\beta \circ \alpha)\left(h \gamma^{c_{1}}\right) \notin(\beta \circ \alpha)(U)$. Set $\psi=\beta \circ \alpha: \operatorname{Ker} \pi=F(Z) \rightarrow B$. Then $\psi(h) \neq \psi\left(\gamma^{a} u z_{0}^{b} u^{-1}\right)$ for all $a, b \in \mathbb{Z}$.

Let $f \in F$ such that $\pi(f)=1$. Let $K=\bigcap_{j=0}^{p^{2}-1}\left(f^{j} \operatorname{Ker} \psi f^{-j}\right)$. The group $K$ is a normal subgroup of $F$ and the quotient $P=F / K$ is a finite $p$-group. Let $\varphi: F \rightarrow P$ be the quotient map. Then $\varphi\left(\gamma^{a} g \gamma^{b} g^{-1}\right) \neq \varphi(h)$ for all $a, b \in \mathbb{Z}$.

Proof of Lemma 4.8. The condition $\gamma^{a} g \neq h \gamma^{b}$ for all $a, b \in \mathbb{Z}$ is equivalent to $\gamma^{a} g \gamma^{-b} g^{-1} \neq h g^{-1}$ for all $a, b \in \mathbb{Z}$. By Lemma 5.2, there exists a homomorphism $\varphi: F \rightarrow P$ such that $P$ is a finite $p$-group and $\varphi\left(\gamma^{a} g \gamma^{-b} g^{-1}\right) \neq \varphi\left(h g^{-1}\right)$ for all $a, b \in \mathbb{Z}$. Then $\varphi\left(\gamma^{a} g\right) \neq \varphi\left(h \gamma^{b}\right)$ for all $a, b \in \mathbb{Z}$.

\section{ACKNOWLEDGMENTS}

The author started this project with Ben McReynolds. Unfortunately, the distance and the activities of both made that the author continued alone. The author hopes he will be forgiven, and thanks him for all the comments and ideas. Most of the results of Sections 2 and 3 are derived from conversations with Laurent Bartholdi. The author thanks him for his significant contribution. Finally, the author thanks Mustafa Korkmaz for suggesting the use of topological arguments in the proof of Theorem 4.1, and for all the conversations on the subject.

\section{REFERENCES}

[1] Travaux de Thurston sur les surfaces. Séminaire Orsay. Astérisque, 66-67. Société Mathématique de France, Paris, 1979. MR568308 (82m:57003)

[2] R. Baer. Isotopie von Kurven auf orientierbaren, geschlossenen Flächen und ihr Zusamen hang mit der topologischen Deformation der Flächen. J. Reine Angew. Math. 159 (1928), 101-116.

[3] H. Bass, A. Lubotzky. Linear-central filtrations on groups. The mathematical legacy of Wilhelm Magnus: groups, geometry and special functions (Brooklyn, NY, 1992), 45-98, Contemp. Math., 169, Amer. Math. Soc., Providence, RI, 1994. MR1292897 (96c:20054)

[4] N. Bourbaki. Groupes et algèbres de Lie. Chapitres II et III. Hermann, Paris, 1972. MR0573068 (58:28083a)

[5] P. Dehornoy, I. Dynnikov, D. Rolfsen, B. Wiest. Why are braids orderable? Panoramas et Synthèses, 14. Société Mathématique de France, Paris, 2002. MR1988550 (2004e:20062)

[6] B. Farb. Some problems on mapping class groups and moduli space. Problems on mapping class groups and related topics, 11-55, Proc. Sympos. Pure Math., 74, Amer. Math. Soc., Providence, RI, 2006. MR2264130(2007h:57018)

[7] E.K. Grossman. On the residual finiteness of certain mapping class groups. J. London Math. Soc. (2) 9 (1974/75), 160-164. MR0405423 (53:9216)

[8] R. Hain. Infinitesimal presentations of the Torelli groups. J. Amer. Math. Soc. 10 (1997), no. 3, 597-651. MR 1431828 (97k:14024)

[9] G. Higman. Amalgams of p-groups. J. Algebra 1 (1964), 301-305. MR0167527 (29:4799)

[10] N.V. Ivanov. Subgroups of Teichmüller modular groups. Translations of Mathematical Monographs, 115. American Mathematical Society, Providence, RI, 1992. MR1195787 (93k:57031)

[11] N.V. Ivanov. Mapping class groups. Handbook of geometric topology, 523-633, NorthHolland, Amsterdam, 2002. MR1886678 (2003h:57022) 
[12] E.A. Ivanova. On the approximability with respect to conjugacy of free products of two groups with an amalgamated subgroup by finite p-groups. (Russian) Mat. Zametki 76 (2004), no. 4, 502-509. Translation in Math. Notes 76 (2004), no. 3-4, 465-471. MR2112066 (2005j:20028)

[13] A. Lubotzky. Normal automorphisms of free groups. J. Algebra 63 (1980), no. 2, 494-498. MR570726 (81k:20041)

[14] R.C. Lyndon, P.E. Schupp. Combinatorial group theory. Reprint of the 1977 edition. Classics in Mathematics. Springer-Verlag, Berlin, 2001. MR,1812024 (2001i:20064)

[15] W. Magnus. Über Automorphismen von Fundamentalgruppen berandeter Flächen. Math. Ann. 109 (1934), 617-646. MR1512913

[16] W. Magnus, A. Karrass, D. Solitar. Combinatorial group theory. Presentations of groups in terms of generators and relations. Second revised edition. Dover Publications, Inc., New York, 1976. MR0422434 (54:10423)

[17] R. Botto Mura, A. Rhemtulla. Orderable groups. Lecture Notes in Pure and Applied Mathematics, Vol. 27. Marcel Dekker, Inc., New York-Basel, 1977. MR0491396 (58:10652)

[18] J. Nielsen. Untersuchungen zur Topologie der geschlossenen zweiseitigen Flächen. Acta Math. 50 (1927), 189-358.

[19] L. Paris. On the fundamental group of the complement of a complex hyperplane arrangement. Arrangements - Tokyo 1998, 257-272, Adv. Stud. Pure Math., 27, Kinokuniya, Tokyo, 2000. MR 1796904 (2001k:32050)

[20] J.-P. Serre. Arbres, amalgames, $S L_{2}$. Astérisque, No. 46. Société Mathématique de France, Paris, 1977. MR0476875 (57:16426)

[21] P.F. Stebe. Conjugacy separability of certain free products with amalgamation. Trans. Amer. Math. Soc. 156 (1971), 119-129. MR0274597 (43:360)

[22] H. Zieschang. Discrete groups of plane motions and plane group images. (Russian) Uspehi Mat. Nauk 21 (1966), no. 3, 195-212. MR0195954 (33:4150)

[23] H. Zieschang, E. Vogt, H.-D. Coldewey. Surfaces and planar discontinuous groups. Lecture Notes in Mathematics, 835. Springer, Berlin, 1980. MR606743 (82h:57002)

Institut de Mathématiques de Bourgogne, UMR 5584 du CNRS, Université de BourGogne, B.P. 47870, 21078 Dijon CEDex, France

E-mail address: lparis@u-bourgogne.fr 